Preprint typeset in JHEP style - HYPER VERSION

McGill-02/31

SLAC-PUB-9511

DAMTP-2002-120

\title{
Low-Energy Brane-World Effective Actions and Partial Supersymmetry Breaking
}

\author{
C. P. Burgess ${ }^{1}$, E. Filotas ${ }^{1}$, M. Klein ${ }^{2}$ and F. Quevedo ${ }^{3}$ \\ 1 Physics Department, McGill University, 3600 University Street, \\ Montréal, Québec, Canada, H3A $2 T 8$. \\ 2 Stanford Linear Accelerator Center, Stanford University, \\ Stanford, CA 94309, USA. \\ 3 Centre for Mathematical Sciences, DAMTP, University of Cambridge, \\ Cambridge CB3 $0 W A$, UK.
}

\begin{abstract}
As part of a programme for the general study of the low-energy implications of supersymmetry breaking in brane-world scenarios, we study the nonlinear realization of supersymmetry which occurs when breaking $N=2$ to $N=1$ supergravity. We consider three explicit realizations of this supersymmetry breaking pattern, which correspond to breaking by one brane, by one antibrane or by two (or more) parallel branes. We derive the minimal field content, the effective action and supersymmetry transformation rules for the resulting $N=1$ theory perturbatively in powers of $\kappa=1 / M_{\text {Planck }}$. We show that the way the massive gravitino and spin-1 fields assemble into $N=1$ multiplets implies the existence of direct brane-brane contact interactions at order $\mathcal{O}(\kappa)$. This result is contrary to the $\mathcal{O}\left(\kappa^{2}\right)$ predicted by the sequestering scenario but in agreement with recent work of Anisimov et al. Our low-energy approach is model independent and is a first step towards determining the low-energy implications of more realistic brane models which completely break all supersymmetries.
\end{abstract}

KEYWORDS: supersymmetry breaking, D-branes. 


\section{Contents}

1. Introduction 1

2. The Single Brane

2.1 The Massive $N=1$ Spin 3/2 Multiplet 5

2.2 UnHiggsing the Massive Spin-3/2 Multiplet 6

2.3 The Nonlinearly Realized Supersymmetry 8

2.4 Coupling to $N=1$ Supergravity 8

2.5 Bulk - Brane Split 11

3. The Single Antibrane 12

3.1 The Brane - Antibrane Map

4. Two Branes 14

4.1 The Massive Spin-1 Multiplet 15

4.2 The Two-Brane System 16

4.3 Noether Coupling to Two Branes 18

5. Conclusions 19

A. Conventions 20

A.1 Majorana spinor formalism 21

A.2 Weyl spinor formalism 22

B. Derivation of Supersymmetry Transformations 24

B.1 The Massive Spin-3/2 Multiplet 24

B.2 The Massive Spin-1 Multiplet 25

\section{Introduction}

String theory is our most promising theory of physics at the highest energies, and predicts that the world is supersymmetric at a very fundamental level. Unfortunately, it does not yet seem to unambiguously predict how this supersymmetry gets broken as it must be in order to make contact with the low-energy world of present-day experiments. An understanding of supersymmetry breaking in string theory appears 
to be a crucial prerequisite for bringing string theory into the mainstream of physical thought.

The discovery of D-branes [1] introduced a fundamentally new mechanism for supersymmetry breaking in string theory, since each D-brane typically breaks half of the supersymmetries of the theory. This mechanism differs from others which are usually considered when model building with supersymmetric field theories, in that it evades [2] an old no-go theorem [3] which was thought to rule out the possibility of partially breaking an extended supersymmetry.

Having the branes themselves break supersymmetry is a particularly appealing picture within a brane-world scenario, for several reasons.

- If the lowest-energy supersymmetry is broken on a brane which is physically separated from the brane on which all observed particles are trapped, then we have a natural realization of the hidden-sector models which have been long recognized as being of great phenomenological interest. Since the interbrane couplings are generically of gravitational strength, the supersymmetry breaking scale is naturally much smaller than the string scale, potentially allowing a novel understanding of the origin of the electroweak hierarchy [⿶].

- 'Realistic' string models having low-energy spectra which contain the known Standard Model fields have been constructed [5], for which all observed particles are trapped on a brane and supersymmetry is broken purely by the configuration of branes considered.

- A framework where supersymmetry partially breaks on distant branes, with the news of this breaking reaching us only through a bulk space which has more supersymmetry than does our brane, has the potential of alleviating some of the naturalness problems which usually plague supersymmetry breaking. Although the consequences of the extra bulk supersymmetries have not yet been explored for the flavor problem of supersymmetry breaking, it has been argued to have the potential to help with the cosmological constant problem [6].

In addition to having a bulk sector which enjoys extended supersymmetry, another very interesting brane-based mechanism has been proposed for suppressing, within supersymmetric models, the direct couplings between fields which live on different branes which are separated from one another in the extra dimensions. According to this mechanism, called sequestering [7], the combination of locality in the extra dimensions with $N=1$ supersymmetry in 4 dimensions suppresses the couplings between fields which live on different branes. For instance, it is proposed that the Kähler potential, $K_{\text {tot }}$, for the total $4 \mathrm{D}$ low-energy theory describing a two-brane configuration, has the form

$$
\exp \left(-\frac{\kappa^{2} K_{\text {tot }}}{3}\right)=\exp \left(-\frac{\kappa^{2} K_{1}}{3}\right)+\exp \left(-\frac{\kappa^{2} K_{2}}{3}\right)
$$


where $K_{1}$ and $K_{2}$ are the Kähler functions describing the $4 \mathrm{D}$ supergravity separately on each of the branes. Here $\kappa^{2}=8 \pi G=1 / M_{p}^{2}$ is the $4 \mathrm{D}$ gravitational coupling constant. If true, this remarkable formula would strongly constrain the kinds of direct couplings which could arise between brane fields, allowing other induced couplings such as anomaly-mediated interactions [7, 8] - to dominate.

The authors of ref. [9] examined this sequestered form in various specific string compactifications, and found that it was not generically satisfied in the string vacua examined. The failure of this form was traced by these authors either to the exchange of various bulk supergravity modes, or to the warping of the compact dimensions.

In this paper we perform a complementary study of sequestering, by directly examining the implications of both the unbroken and the nonlinearly-realized supersymmetries for the low-energy $4 \mathrm{D}$ effective action for any brane model which spontaneously breaks $N=2$ supersymmetry to $N=1$ supersymmetry. We show that the way the massive gravitino and spin- 1 fields assemble into $N=1$ supersymmetry multiplets implies the existence of direct brane-brane contact interactions already at $\mathcal{O}(\kappa)$, in conflict with the $\mathcal{O}\left(\kappa^{2}\right)$ size which would be predicted by eq. (1.1). In this way we show that the failure of the sequestered form is quite robust, and cannot hold in any brane configuration which breaks $N=2$ supersymmetry down to $N=1$. We believe it to be an open question whether sequestering can occur in realistic brane-world models, and we discuss some evidence that it might require additional supersymmetries in the concluding section.

More generally, our investigation can be seen as a first step towards the study of the low-energy limit of more realistic models for which supersymmetry is completely broken by brane configurations situated within bulk spacetimes enjoying extended supersymmetries. Indeed this is our prime motivation for embarking on this programme, and we consider it as the natural next step in this direction. In particular, if brane supersymmetry breaking is to play a role in solving naturalness problems at low energies, it should be possible to understand how this is done purely within the effective theory below the compactification scale, within which all the information about brane separations has already been integrated out. For this reason we cast our discussion completely in terms of how supersymmetry is realized within the lowenergy four-dimensional theory. (For recent discussions of supersymmetry breaking in the brane-world scenario from a more microscopic perspective see, for instance, [10].)

The questions which such a study of low-energy supersymmetry breaking could hope to address include: finding the effective scale of mass splittings within supermultiplets, as compared to the string and compactification scales; the structure of softand hard-breaking interactions, with a comparison with the standard $F$ - and $D$-term breaking in more standard supergravity-mediated models; the consequences of extended supersymmetries for gauge- and anomaly-mediated supersymmetry breaking; the relative suppression of the remnant value of the cosmological constant as com- 
pared to the scale of multiplet splitting. (First steps along some of these lines have been made for $5 \mathrm{D}$ theories, such as by establishing the connection between $F$-term supersymmetry breaking in the low-energy theory and Scherk-Schwarz supersymmetry breaking in microscopic brane models [11].) A proper study of all of these issues lies at the core of any understanding of the phenomenological implications of D-brane models.

Our presentation is organized as follows. First we describe how the low-energy states associated with the gravitini are organized in a model which breaks $N=2$ supersymmetry down to $N=1$ in four spacetime dimensions. The generalization of this result to an antibrane is also briefly described. We then extend this analysis to the case where more than two branes participate in breaking the one supersymmetry, and so implying that the would-be Goldstone modes are a mixture of fields which arise on the two branes. In this case we show how the assembly of states into supermultiplets of the unbroken $N=1$ supersymmetry require the existence of direct brane couplings at $\mathcal{O}(\kappa)$. Our conclusions are briefly summarized in the final section. A warning about notation: we present our results using the 4-component spinor notation which is commonly used outside of the supersymmetry community. However, for the benefit of the reader we also present in an appendix a dictionary which makes the explicit connection with two-component Weyl-spinor notation. Further technical details on transformation rules for massive spin $3 / 2$ and spin 1 are presented in a second appendix.

\section{The Single Brane}

Imagine now any configuration of branes within a higher-dimensional spacetime that is compactified to flat four dimensions, for which a $4 \mathrm{D} N=2$ supersymmetry is unbroken. Suppose also that the brane configuration breaks this $N=2$ supersymmetry, leaving an $N=1$ supersymmetry unbroken [12].

Our purpose in this section is to describe the universal part of the low-energy limit of such a configuration, focussing on those very low energy degrees of freedom whose mass is well below the model's compactification scale $E \ll 1 / r$. The goal is to identify general features of the $4 \mathrm{D}$ effective theory defined after all of the extra-dimensional physics is integrated out, to see how the supersymmetry-breaking pattern is realized in the low-energy theory [13, 14]. Our analysis closely follows the pioneering work of ref. [14], although we do find a few minor corrections to some of the results found in that paper.

Following ref. [14], we obtain the low-energy theory by using the following two properties which the spectrum of any such brane construction enjoys.

- First, the spectrum necessarily involves two spin-3/2 gravitini. One of these is massless (for the unbroken supersymmetry) while the other is massive (for 
the broken supersymmetry). Although massive, this second gravitino is nevertheless within the low-energy theory because its mass can be well below the compactification scale, $1 / r$.

- Second, the unbroken supersymmetry organizes these two gravitini into $N=1$ supermultiplets, with the massless gravitino being paired with the graviton and the massive gravitino combining with two massive spin- 1 and one massive spin-1/2 particle into a massive $N=1$ spin-3/2 multiplet.

The strategy is to identify the brane and bulk degrees of freedom by writing down the lagrangian for these two gravitino multiplets, and then unHiggsing the massive spin-1 and spin-3/2 fields [14] to identify the would-be Goldstone modes which are eaten to produce these massive states.

\subsection{The Massive $N=1$ Spin 3/2 Multiplet}

Our starting point is the free lagrangian for a massive spin-3/2 multiplet in $N=1$ global supersymmetry. This multiplet must contain two massive spin-1 $\left(\mathcal{A}_{m}=\left(A_{m}+\right.\right.$

$\left.i B_{m}\right) / \sqrt{2}$ ) and one massive spin-1/2 particle $(\zeta)$ as well as the massive spin-3/2 field $\left(\eta_{m}\right)$. Its free action may therefore be written:

$$
\begin{array}{r}
\mathcal{L}_{\text {kin }}=-\frac{i}{2} \epsilon^{m n p q} \bar{\eta}_{m} \gamma_{5} \gamma_{n} \partial_{p} \eta_{q}-\frac{1}{2} \mathcal{A}^{m n} \mathcal{A}_{m n}^{*}-\frac{1}{2} \bar{\zeta} \not \partial \zeta \\
-\frac{m}{2} \bar{\eta}_{m} \gamma^{m n} \eta_{n}-m^{2} \mathcal{A}^{m} \mathcal{A}_{m}^{*}-\frac{m}{2} \bar{\zeta} \zeta
\end{array}
$$

where $\mathcal{A}_{m n}=\partial_{m} \mathcal{A}_{n}-\partial_{n} \mathcal{A}_{m}$ satisfies $\epsilon^{m n p q} \partial_{n} \mathcal{A}_{p q}=0$. In our conventions the fermionic fields, $\zeta$ and $\eta_{m}$, are chosen to be Majorana spinors. The freedom to rescale the fields has been used to put all kinetic terms into canonical form.

As is easily verified, this action enjoys an invariance with respect to the following global supersymmetry transformations [15]:

$$
\begin{aligned}
\delta \mathcal{A}_{m}= & \sqrt{2}\left(\bar{\eta}_{m} \gamma_{L} \varepsilon\right)+\frac{2}{\sqrt{6}}\left(\bar{\zeta} \gamma_{m} \gamma_{L} \varepsilon\right)-\frac{2}{\sqrt{6} m} \partial_{m}\left(\bar{\zeta} \gamma_{L} \varepsilon\right) \\
\gamma_{L} \delta \zeta= & \frac{1}{\sqrt{6}} \mathcal{A}_{m n}^{*} \gamma^{m n} \gamma_{L} \varepsilon-\frac{2 m}{\sqrt{6}} \mathcal{A}_{m} \gamma^{m} \gamma_{R} \varepsilon \\
\gamma_{L} \delta \eta_{m}= & \frac{1}{m} \partial_{m}\left(\frac{\sqrt{2}}{6} \mathcal{A}_{a b}^{*} \gamma^{a b} \gamma_{L} \varepsilon+\frac{2 \sqrt{2} m}{3} \mathcal{A}_{a} \gamma^{a} \gamma_{R} \varepsilon\right)-\frac{2 \sqrt{2}}{3} \mathcal{A}_{m n} \gamma^{n} \gamma_{R} \varepsilon \\
& +\frac{i \sqrt{2}}{6} \epsilon_{m n a b} \mathcal{A}^{a b} \gamma^{n} \gamma_{R} \varepsilon-\frac{\sqrt{2} m}{3} \mathcal{A}^{n *} \gamma_{m n} \gamma_{L} \varepsilon+\frac{2 \sqrt{2} m}{3} \mathcal{A}_{m}^{*} \gamma_{L} \varepsilon
\end{aligned}
$$

where $\varepsilon$ denotes the Majorana supersymmetry transformation parameter. 


\subsection{UnHiggsing the Massive Spin-3/2 Multiplet}

The transformation laws, (2.2), appear to be singular in the massless limit, $m \rightarrow 0$. This is an artifact of our use of massive fields in this expression - which amounts to the use of unitary gauge for the broken supersymmetry and the broken gauge invariance. The apparent singularity is removed by unHiggsing this system: that is, by switching to a more convenient gauge by explicitly identifying the wouldbe Goldstone modes whose consumption produced the massive spin- 1 and spin-3/2 fields. On general grounds such a transformation is always possible [16, 17], despite the apparent lack of gauge invariance of the system.

To perform the unHiggsing we shift the fields $\mathcal{A}_{m}$ and $\eta_{m}$ to expose the eaten scalar, $\phi$, and the eaten goldstino, $\xi$, as follows:

$$
\begin{aligned}
\mathcal{A}_{m} & \rightarrow \mathcal{A}_{m}^{\prime}=\mathcal{A}_{m}-\frac{a}{m} \partial_{m} \phi \\
\gamma_{L} \eta_{m} \rightarrow \gamma_{L} \eta_{m}^{\prime} & =\gamma_{L}\left[\eta_{m}+\frac{b}{m} \partial_{m} \xi+c \gamma_{m} \xi\right] .
\end{aligned}
$$

The complex constants $a, b, c$ are determined by requiring that the transformation does not generate off-diagonal kinetic terms in the lagrangian which mix $\eta_{m}$ and $\xi$, or $\mathcal{A}_{m}$ and $\phi$, and by requiring the new fields $\phi$ and $\xi$ to have canonical kinetic terms. These requirements are satisfied by the choice $a=1, b=2 i / \sqrt{6}$ and $c=i / \sqrt{6}$, and so we have:

$$
\begin{aligned}
\mathcal{A}_{m}^{\prime} & =\mathcal{A}_{m}-\frac{1}{m} \partial_{m} \phi=-\frac{1}{m} \mathcal{D}_{m} \phi, \\
\eta_{m}^{\prime} & =\eta_{m}+\frac{2 i}{\sqrt{6} m} \gamma_{5} \partial_{m} \xi+\frac{i}{\sqrt{6}} \gamma_{5} \gamma_{m} \xi .
\end{aligned}
$$

The second equality in the first line of these equations defines the scalar covariant derivative $\mathcal{D}_{m} \phi=\partial_{m} \phi-m \mathcal{A}_{m}$.

With these choices the lagrangian, eq. (2.1), becomes:

$$
\begin{gathered}
\mathcal{L}=-\frac{i}{2} \epsilon^{m n p q} \bar{\eta}_{m} \gamma_{5} \gamma_{n} \partial_{p} \eta_{q}-\frac{1}{2} \mathcal{A}_{m n}^{*} \mathcal{A}^{m n}-\frac{1}{2} \bar{\zeta} \not \partial \zeta-\frac{1}{2} \bar{\xi} \not \partial \xi-\mathcal{D}_{m} \phi^{*} \mathcal{D}^{m} \phi \\
-\frac{m}{2} \bar{\eta}_{m} \gamma^{m n} \eta_{n}-\frac{m}{2} \bar{\zeta} \zeta-m \bar{\xi} \xi-i m \sqrt{\frac{3}{2}}\left(\bar{\eta}_{m} \gamma_{5} \gamma^{m} \xi\right) .
\end{gathered}
$$

We next define the supersymmetry transformations for the new fields $\phi$ and $\xi$. This is done by partitioning the supersymmetry transformations of $\mathcal{A}_{m}^{\prime}$ and $\eta_{m}^{\prime}$ amongst the fields $\mathcal{A}_{m}, \phi, \eta_{m}$ and $\xi$ in such a way as to ensure that there are no terms proportional to $1 / m$. For $\phi$ this gives:

$$
\delta \phi=i \sqrt{2} \bar{\varepsilon} \gamma_{L}\left[\frac{1}{\sqrt{3}}(-i \zeta-\sqrt{2} \xi)\right],
$$


which states that the left-handed part of the combination

$$
\chi=\frac{1}{\sqrt{3}}\left(-i \gamma_{5} \zeta-\sqrt{2} \xi\right)
$$

transforms with $\phi$ with the standard form for $\delta \phi$ of a left-chiral multiplet [18]. The orthogonal combination we define as

$$
\lambda=\frac{1}{\sqrt{3}}\left(-\sqrt{2} \zeta-i \gamma_{5} \xi\right) .
$$

We similarly choose the transformation rules for $\xi$ to ensure the absence of $1 / \mathrm{m}$ terms in $\delta \eta_{m}$. We find we must choose:

$$
\gamma_{L} \delta \xi=-\frac{i}{2 \sqrt{3}} \mathcal{A}_{m n}^{*} \gamma^{m n} \gamma_{L} \varepsilon+\frac{2 i}{\sqrt{3}} \mathcal{D}_{m} \phi \gamma^{m} \gamma_{R} \varepsilon
$$

in which case $\lambda$ and $\chi$ transform according to:

$$
\begin{aligned}
& \gamma_{L} \delta \chi=-i \sqrt{2} \mathcal{D}_{m} \phi \gamma^{m} \gamma_{R} \varepsilon \\
& \gamma_{L} \delta \lambda=-\frac{1}{2} \mathcal{A}_{m n}^{*} \gamma^{m n} \gamma_{L} \varepsilon
\end{aligned}
$$

Notice that $\mathcal{A}_{m}$ does not appear in $\delta \chi$ and that $\phi$ drops out of $\delta \lambda$. This amounts to the statement that $\chi$ and $\phi$ form a standard $N=1$ chiral multiplet and $\lambda$ and $A_{m}$ form an $N=1$ gauge multiplet.

With these choices we may also write down the transformation law for $\mathcal{A}_{m}$ and $\eta_{m}$. They are:

$$
\begin{aligned}
\delta \phi & =i \sqrt{2} \bar{\varepsilon} \gamma_{L} \chi \\
\gamma_{L} \delta \chi & =-i \sqrt{2} \mathcal{D}_{m} \phi \gamma^{m} \gamma_{R} \varepsilon \\
\gamma_{L} \delta \lambda & =-\frac{1}{2} \mathcal{A}_{m n}^{*} \gamma^{m n} \gamma_{L} \varepsilon \\
\delta \mathcal{A}_{m} & =\sqrt{2} \bar{\eta}_{m} \gamma_{L} \varepsilon+\bar{\varepsilon} \gamma_{m} \gamma_{R} \lambda \\
\gamma_{L} \delta \eta_{m} & =-\frac{1}{\sqrt{2}} \mathcal{A}_{m n}^{-} \gamma^{n} \gamma_{R} \varepsilon-\sqrt{2} \mathcal{D}_{m} \phi^{*} \gamma_{L} \varepsilon
\end{aligned}
$$

where $\mathcal{A}_{m n}^{-}=\mathcal{A}_{m n}-\frac{i}{2} \epsilon_{m n a b} \mathcal{A}^{a b}$. The lagrangian in these variables becomes:

$$
\begin{aligned}
\mathcal{L}= & -\frac{i}{2} \epsilon^{m n p q} \bar{\eta}_{m} \gamma_{5} \gamma_{n} \partial_{p} \eta_{q}-\frac{1}{2} \mathcal{A}_{m n}^{*} \mathcal{A}^{m n}-\frac{1}{2} \bar{\chi} \not \partial \chi-\frac{1}{2} \bar{\lambda} \not \partial \lambda-\mathcal{D}_{m} \phi^{*} \mathcal{D}^{m} \phi \\
& -\frac{m}{2} \bar{\eta}_{m} \gamma^{m n} \eta_{n}-\frac{m}{2} \bar{\chi} \chi+i \sqrt{2} m \bar{\lambda} \gamma_{5} \chi+i m \bar{\eta}_{m} \gamma_{5} \gamma^{m} \chi-\frac{m}{\sqrt{2}} \bar{\eta}_{m} \gamma^{m} \lambda .
\end{aligned}
$$

Notice that both the transformations, eq. (2.16), and lagrangian density, eq. (2.17), now have perfectly smooth limits as $m \rightarrow 0$. Notice also that the fermion mass terms also have the usual form, with no diagonal gaugino-gaugino pieces, but only $\chi-\lambda$ mixing plus a $\chi$ mass term. 


\subsection{The Nonlinearly Realized Supersymmetry}

We may now identify the second, nonlinearly-realized, supersymmetry of the action, eq. (2.17). Since the argument closely parallels that of the nonlinearly-realized internal $U(1) \times U(1)$ gauge invariance of the massive spin-1 particles, we first examine this simpler case.

The nonlinearly-realized $U(1) \times U(1)$ gauge invariance simply expresses the fact that the two complex fields $\mathcal{A}_{m}$ and $\phi$ only enter the lagrangian through the single combination $\mathcal{A}_{m}^{\prime}=\mathcal{A}_{m}-\partial_{m} \phi / m$ [16]. The symmetry therefore is

$$
\delta \phi=m \omega(x), \quad \delta \mathcal{A}_{m}=\partial_{m} \omega,
$$

where the transformation parameter, $\omega$, is normalized to ensure $\delta \mathcal{A}_{m}$ transforms in a conventional way.

The nonlinearly-realized supersymmetry transformation similarly expresses the fact that the fields $\xi$ and $\eta_{m}$ only appear through the single combination $\eta_{m}^{\prime}$. Adopting a conventional normalization for the transformation parameter we therefore have the second supersymmetry of eq. (2.17):

$$
\delta \xi=\frac{i \sqrt{6} m}{\kappa} \gamma_{5} \eta, \quad \delta \eta_{m}=\frac{2}{\kappa} \partial_{m} \eta-\frac{m}{\kappa} \gamma_{m} \eta .
$$

This transformation rule for $\xi$ implies $\chi$ and $\lambda$ transform according to

$$
\delta \lambda=\frac{\sqrt{2} m}{\kappa} \eta, \quad \delta \chi=-\frac{2 i m}{\kappa} \gamma_{5} \eta .
$$

No other fields transform (to this order in $\kappa$ ) under this supersymmetry.

\subsection{Coupling to $N=1$ Supergravity}

Given the globally supersymmetric action just described, we may immediately write down the coupling to the massless spin- $3 / 2$ and spin- 2 fields. This is accomplished using standard methods by coupling to $N=1$ supergravity. Our interest in what follows is only in those couplings which arise at lowest order in powers of $\kappa$.

The fields in the gravity multiplet are the vierbein, $e_{m}{ }^{a}$, and massless gravitino, $\psi_{m}$. The lagrangian density for these fields is:

$$
\mathcal{L}_{s g}=-\frac{e}{2 \kappa^{2}} R-\frac{i}{2} \epsilon^{m n p q} \bar{\psi}_{m} \gamma_{5} \gamma_{n} D_{p} \psi_{q},
$$

which is invariant with respect to the local supersymmetry transformation:

$$
\delta e_{m}{ }^{a}=\kappa \bar{\varepsilon} \gamma^{a} \psi_{m}, \quad \delta \psi_{m}=\frac{2}{\kappa} D_{m} \varepsilon,
$$

where $D_{m} \varepsilon=\partial_{m} \varepsilon+\frac{1}{4} \omega_{m}^{a b} \gamma_{a b} \varepsilon$. The spin connection, $\omega_{m}^{a b}$, contains gravitino torsion at order $\kappa^{2}$, but this is negligible to the order in $\kappa$ to which we work in $\mathcal{L}$. In what 
follows we may therefore treat $D_{m}$ as if it had no torsion terms, allowing us to treat the vierbein and Dirac matrices as if they were covariantly constant. As usual we denote the vierbein determinant by $e=\operatorname{det}\left(e_{m}{ }^{a}\right)$.

The Noether prescription [19] can be used to obtain leading order couplings between the massless $\left(2, \frac{3}{2}\right)$ multiplet and the massive $\left(\frac{3}{2}, 1,1, \frac{1}{2}\right)$ multiplet [15]. The starting point for this method is the observation that any globally supersymmetric action transforms under local supersymmetry as:

$$
\delta \mathcal{L}=e\left(D_{m} \bar{\varepsilon}\right) U^{m}+(\text { total derivative }),
$$

for some Majorana spinor-vector $U^{m}$. The derivative appearing here is covariant because we imagine having also already made $\mathcal{L}$ generally covariant by replacing all derivatives in the globally supersymmetric action by covariant derivatives. To $\mathcal{O}\left(\kappa^{0}\right)$ this variation is cancelled by the Noether coupling: $\mathcal{L}_{\kappa}=-\frac{\kappa}{2} e \bar{\psi}_{m} U^{m}$, due to the $\mathcal{O}(1 / \kappa)$ variation $\delta \psi_{m}=(2 / \kappa) D_{m} \varepsilon$.

Directly varying the lagrangian density, eq. (2.17), we find:

$$
\begin{aligned}
\delta \mathcal{L}= & (\text { total derivative })+i \sqrt{2}\left(D_{n} \bar{\varepsilon} \gamma^{m} \gamma^{n} \gamma_{R} \chi\right) \mathcal{D}_{m} \phi-\mathcal{A}_{+}^{m n}\left(D_{m} \bar{\varepsilon} \gamma_{n} \gamma_{L} \lambda\right) \\
& +i \sqrt{2} \epsilon^{m n p q}\left(D_{m} \bar{\varepsilon} \gamma_{n} \gamma_{L} \eta_{p}\right) \mathcal{D}_{q} \phi+\frac{i}{\sqrt{2}} \epsilon^{m n p q}\left(D_{m} \bar{\varepsilon} \gamma^{r} \gamma_{n} \gamma_{R} \eta_{p}\right) \mathcal{A}_{q r}^{-}+\text {c.c. }
\end{aligned}
$$

We read off from this the supercurrent, $U^{m}$, for the massive spin-3/2 multiplet:

$$
\begin{aligned}
\gamma_{R} U^{m}= & i \sqrt{2}\left(\gamma^{n} \gamma^{m} \gamma_{R} \chi\right) \mathcal{D}_{n} \phi-\mathcal{A}_{+}^{m n}\left(\gamma_{n} \gamma_{L} \lambda\right) \\
& +i \sqrt{2} \epsilon^{m n p q}\left(\gamma_{n} \gamma_{L} \eta_{p}\right) \mathcal{D}_{q} \phi+\frac{i}{\sqrt{2}} \epsilon^{m n p q}\left(\gamma^{r} \gamma_{n} \gamma_{R} \eta_{p}\right) \mathcal{A}_{q r}^{-} .
\end{aligned}
$$

Here $\mathcal{A}_{m n}^{ \pm}=\mathcal{A}_{m n} \pm \frac{i}{2} \epsilon_{m n p q} \mathcal{A}^{p q}$. This gives the lagrangian to this order to be $\mathcal{L}_{0}+\mathcal{L}_{\kappa}$ where $\mathcal{L}_{0}$ contains the kinetic and mass terms:

$$
\begin{aligned}
\frac{\mathcal{L}_{0}}{e}= & -\frac{1}{2 \kappa^{2}} R-\frac{i}{2 e} \epsilon^{m n p q} \bar{\psi}_{m} \gamma_{5} \gamma_{n} D_{p} \psi_{q}-\frac{i}{2 e} \epsilon^{m n p q} \bar{\eta}_{m} \gamma_{5} \gamma_{n} D_{p} \eta_{q} \\
& -\frac{1}{2} \mathcal{A}_{m n}^{*} \mathcal{A}^{m n}-\frac{1}{2} \bar{\chi} \not \partial-\frac{1}{2} \bar{\lambda} \not \partial \lambda-\mathcal{D}_{m} \phi^{*} \mathcal{D}^{m} \phi \\
& -\frac{m}{2}\left(\bar{\eta}_{m} \gamma^{m n} \eta_{n}\right)-\frac{m}{2} \bar{\chi} \chi+i \sqrt{2} m\left(\bar{\lambda} \gamma_{5} \chi\right)+i m\left(\bar{\eta}_{m} \gamma_{5} \gamma^{m} \chi\right)-\frac{m}{\sqrt{2}}\left(\bar{\eta}_{m} \gamma^{m} \lambda\right)
\end{aligned}
$$

The $\mathcal{O}(\kappa)$ couplings from the Noether prescription are:

$$
\begin{aligned}
\frac{\mathcal{L}_{\kappa}}{e}= & -\frac{i \kappa}{\sqrt{2}}\left(\bar{\psi}_{m} \gamma^{n} \gamma^{m} \gamma_{R} \chi\right) \mathcal{D}_{n} \phi+\frac{\kappa}{2} \mathcal{A}_{+}^{m n}\left(\bar{\psi}_{m} \gamma_{n} \gamma_{L} \lambda\right) \\
& -\frac{i \kappa}{\sqrt{2} e} \epsilon^{m n p q}\left(\bar{\psi}_{m} \gamma_{n} \gamma_{L} \eta_{p}\right) \mathcal{D}_{q} \phi-\frac{i \kappa}{2 \sqrt{2} e} \epsilon^{m n p q}\left(\bar{\psi}_{m} \gamma^{r} \gamma_{n} \gamma_{R} \eta_{p}\right) \mathcal{A}_{q r}^{-}+\text {c.c. }
\end{aligned}
$$

The last term in this expression can be simplified to become:

$$
\begin{aligned}
\frac{\mathcal{L}_{\kappa}}{e}= & -\frac{i \kappa}{\sqrt{2}}\left(\bar{\psi}_{m} \gamma^{n} \gamma^{m} \gamma_{R} \chi\right) \mathcal{D}_{n} \phi+\frac{\kappa}{2} \mathcal{A}_{+}^{m n}\left(\bar{\psi}_{m} \gamma_{n} \gamma_{L} \lambda\right) \\
& -\frac{i \kappa}{\sqrt{2} e} \epsilon^{m n p q}\left(\bar{\psi}_{m} \gamma_{n} \gamma_{L} \eta_{p}\right) \mathcal{D}_{q} \phi+\frac{\kappa}{\sqrt{2}}\left(\bar{\psi}_{m} \gamma_{R} \eta_{n}\right) \mathcal{A}_{-}^{m n}+\text { c.c. }
\end{aligned}
$$


The action $\mathcal{L}=\mathcal{L}_{0}+\mathcal{L}_{\kappa}$ is, by construction, invariant to $\mathcal{O}\left(\kappa^{0}\right)$ under the unbroken supersymmetry. Invariance to the same order in $\kappa$ with respect to the nonlinearly-realized supersymmetry is not automatic, however, since $\mathcal{L}_{\kappa}$ does not depend on the fields $\phi, \mathcal{A}_{m}, \xi$ and $\eta_{m}$ only through the invariant combinations $\mathcal{A}_{m}^{\prime}$ and $\eta_{m}^{\prime}$. Furthermore, since the transformation rules, eq. (2.19), contain terms which are $\mathcal{O}(1 / \kappa)$, the non-invariance of $\mathcal{L}_{\kappa}$ already arises at $\mathcal{O}\left(\kappa^{0}\right)$ in $\delta \mathcal{L}$. We now show that this non-invariance may be cancelled by modifying the transformation rules for some of the fields at $\mathcal{O}\left(\kappa^{0}\right){ }^{1}$

One source of non-invariance is the replacement of ordinary with covariant derivatives in the globally supersymmetric action and transformation rules for the second supersymmetry. This ruins the automatic invariance of the kinetic term for $\eta_{m}$ to this order, since the commutator of the two derivatives acting on $\eta$ is proportional to the Riemann tensor, and so must cancel the variation of the Einstein term. This requires us to add an $\mathcal{O}(\kappa)$ term to $\delta e_{m}{ }^{a}$ involving $\eta_{m}$, to reproduce the cancellation of the $\mathcal{O}(1 / \kappa)$ terms which occurs between the Einstein term and the $\psi_{m}$ kinetic term. The new transformation for the vierbein is then symmetric in the two gravitini:

$$
\delta e_{m}^{a}=\kappa\left(\bar{\varepsilon} \gamma^{a} \psi_{m}+\bar{\eta} \gamma^{a} \eta_{m}\right)
$$

In order to cancel the other $\mathcal{O}\left(\kappa^{0}\right)$ terms in $\delta \mathcal{L}$ we try the following ansatz, which is the most general consistent with Lorentz invariance, dimensional analysis and the $U_{R}(1)$ symmetry:

$$
\begin{aligned}
\gamma_{L} \delta \psi_{m}= & \gamma_{L}\left(\delta_{\text {old }} \psi_{m}\right)+a_{1}\left(\gamma_{L} \eta\right) \mathcal{D}_{m} \phi \\
& \quad+a_{2} \mathcal{A}_{m n}^{+}\left(\gamma^{n} \gamma_{R} \eta\right)+a_{3} \mathcal{A}_{m n}^{-}\left(\gamma^{n} \gamma_{R} \eta\right) \\
\delta \mathcal{A}_{m}= & \left(\delta_{\text {old }} \mathcal{A}_{m}\right)+a_{4}\left(\bar{\psi}_{m} \gamma_{L} \eta\right) \\
\delta \phi= & \left(\delta_{\text {old }} \phi\right)+a_{5}\left(\bar{\psi}_{m} \gamma^{m} \gamma_{R} \eta\right) .
\end{aligned}
$$

The constants $a_{1}, \ldots, a_{5}$ are to be chosen to ensure invariance of $\mathcal{L}$ up to $\mathcal{O}\left(\kappa^{0}\right)$. The choice which does so is:

$$
a_{1}=\sqrt{2}, \quad a_{2}=0, \quad a_{3}=\frac{1}{\sqrt{2}}, \quad a_{4}=-\sqrt{2}, \quad a_{5}=0 .
$$

The combined supersymmetry transformations then are:

$$
\begin{aligned}
\delta \phi & =i \sqrt{2} \bar{\varepsilon} \gamma_{L} \chi \\
\gamma_{L} \delta \chi & =-i \sqrt{2} \mathcal{D}_{m} \phi\left(\gamma^{m} \gamma_{R} \varepsilon\right)-2 i \mu^{2}\left(\gamma_{L} \eta\right) \\
\gamma_{L} \delta \lambda & =-\frac{1}{2} \mathcal{A}_{m n}^{*}\left(\gamma^{m n} \gamma_{L} \varepsilon\right)+\sqrt{2} \mu^{2}\left(\gamma_{L} \eta\right) \\
\delta \mathcal{A}_{m} & =\bar{\varepsilon} \gamma_{m} \gamma_{R} \lambda+\sqrt{2}\left(\bar{\eta}_{m} \gamma_{L} \varepsilon-\bar{\psi}_{m} \gamma_{L} \eta\right)
\end{aligned}
$$

\footnotetext{
${ }^{1}$ It is with the addition of these terms that our results differ from those of ref. [14.
} 


$$
\begin{aligned}
\gamma_{L} \delta \eta_{m}= & \frac{2}{\kappa} D_{m}\left(\gamma_{L} \eta\right)-\mu^{2} \gamma_{m}\left(\gamma_{R} \eta\right) \\
& -\frac{1}{\sqrt{2}} \mathcal{A}_{m n}^{-}\left(\gamma^{n} \gamma_{R} \varepsilon\right)-\sqrt{2} \mathcal{D}_{m} \phi^{*}\left(\gamma_{L} \varepsilon\right) \\
\gamma_{L} \delta \psi_{m}= & \frac{2}{\kappa} D_{m}\left(\gamma_{L} \varepsilon\right)+\frac{1}{\sqrt{2}} \mathcal{A}_{m n}^{-}\left(\gamma^{n} \gamma_{R} \eta\right)+\sqrt{2} \mathcal{D}_{m} \phi\left(\gamma_{L} \eta\right) \\
\delta e_{m}{ }^{a}= & \kappa\left(\bar{\varepsilon} \gamma^{a} \psi_{m}+\bar{\eta} \gamma^{a} \eta_{m}\right),
\end{aligned}
$$

where $\mu^{2}=m / \kappa$.

Eqs. (2.25), (2.27) and (2.31) are the main results of this section, and are generic to the low-energy limit of any brane configuration for which supersymmetry is partially broken from $N=2$ to $N=1$ in four dimensions.

\subsection{Bulk - Brane Split}

Before proceeding, for later purposes it is worth splitting the fields into two categories which, microscopically, correspond to those which live on a brane and those which live in the bulk. This split can be made macroscopically by separating out that part of the lagrangian which enjoys unbroken $N=2$ supersymmetry, and assigning this to the bulk.

In order to do so in the present instance we assign to the bulk the members of the massless $N=2$ supergravity multiplet: $\left\{e^{a}{ }_{m}, \psi_{m}, \eta_{m}, B_{m}\right\}$. The bulk action consists of those terms considered previously which depend only on these fields, and which do not depend on the supersymmetry-breaking scale $m$ (or $\mu$ ). Keeping in mind $\mathcal{A}_{m}=\left(A_{m}+i B_{m}\right) / \sqrt{2}$, we find:

$$
\begin{gathered}
\frac{\mathcal{L}_{B}}{e}=-\frac{1}{2 \kappa^{2}} R-\frac{i}{2 e} \epsilon^{m n p q} \bar{\psi}_{m} \gamma_{5} \gamma_{n} D_{p} \psi_{q}-\frac{i}{2 e} \epsilon^{m n p q} \bar{\eta}_{m} \gamma_{5} \gamma_{n} D_{p} \eta_{q} \\
-\frac{1}{4} B_{m n} B^{m n}+\left[\frac{i \kappa}{2}\left(\bar{\psi}_{m} \gamma_{R} \eta_{n}\right) B_{-}^{m n}+\text { c.c. }\right] .
\end{gathered}
$$

Eq. (2.32) is invariant under the restriction of the supersymmetry transformations to these fields, which we call the bulk part of the transformations:

$$
\begin{aligned}
\delta_{B} B_{m} & =-i\left(\bar{\eta}_{m} \gamma_{5} \varepsilon-\bar{\psi}_{m} \gamma_{5} \eta\right) \\
\gamma_{L} \delta_{B} \eta_{m} & =\frac{2}{\kappa} D_{m}\left(\gamma_{L} \eta\right)-\frac{i}{2} B_{m n}^{-}\left(\gamma^{n} \gamma_{R} \varepsilon\right) \\
\gamma_{L} \delta_{B} \psi_{m} & =\frac{2}{\kappa} D_{m}\left(\gamma_{L} \varepsilon\right)+\frac{i}{2} B_{m n}^{-}\left(\gamma^{n} \gamma_{R} \eta\right) \\
\delta_{B} e_{m}{ }^{a} & =\kappa\left(\bar{\varepsilon} \gamma^{a} \psi_{m}+\bar{\eta} \gamma^{a} \eta_{m}\right) .
\end{aligned}
$$

All of the remaining terms in the action and supersymmetry transformations we lump together as the brane contributions, and so are given to this order in $\kappa$ by

$$
\frac{\mathcal{L}_{b}}{e}=-\frac{1}{4} A_{m n} A^{m n}-\frac{1}{2} \bar{\chi} \not \partial \chi-\frac{1}{2} \bar{\lambda} \not \partial \lambda-\mathcal{D}_{m} \phi^{*} \mathcal{D}^{m} \phi-\frac{m}{2}\left(\bar{\eta}_{m} \gamma^{m n} \eta_{n}\right)
$$




$$
\begin{aligned}
& -\frac{m}{2}(\bar{\chi} \chi)+i \sqrt{2} m\left(\bar{\lambda} \gamma_{5} \chi\right)+i m\left(\bar{\eta}_{m} \gamma_{5} \gamma^{m} \chi\right)-\frac{m}{\sqrt{2}}\left(\bar{\eta}_{m} \gamma^{m} \lambda\right) \\
& -\frac{i \kappa}{\sqrt{2}}\left(\bar{\psi}_{m} \gamma^{n} \gamma^{m} \gamma_{R} \chi\right) \mathcal{D}_{n} \phi+\frac{\kappa}{2} \mathcal{A}_{+}^{m n}\left(\bar{\psi}_{m} \gamma_{n} \gamma_{L} \lambda\right) \\
& -\frac{i \kappa}{\sqrt{2} e} \epsilon^{m n p q}\left(\bar{\psi}_{m} \gamma_{n} \gamma_{L} \eta_{p}\right) \mathcal{D}_{q} \phi+\frac{\kappa}{2}\left(\bar{\psi}_{m} \gamma_{R} \eta_{n}\right) A_{-}^{m n}+\text { c.c. }
\end{aligned}
$$

and

$$
\begin{aligned}
\delta_{b} \phi & =i \sqrt{2} \bar{\varepsilon} \gamma_{L} \chi \\
\gamma_{L} \delta_{b} \chi & =-i \sqrt{2} \mathcal{D}_{m} \phi\left(\gamma^{m} \gamma_{R} \varepsilon\right)-2 i \mu^{2}\left(\gamma_{L} \eta\right) \\
\gamma_{L} \delta_{b} \lambda & =-\frac{1}{2} \mathcal{A}_{m n}^{*}\left(\gamma^{m n} \gamma_{L} \varepsilon\right)+\sqrt{2} \mu^{2}\left(\gamma_{L} \eta\right) \\
\delta_{b} A_{m} & =\frac{1}{\sqrt{2}}\left(\bar{\varepsilon} \gamma_{m} \lambda\right)+\left(\bar{\eta}_{m} \varepsilon-\bar{\psi}_{m} \eta\right) \\
\delta_{b} B_{m} & =\frac{i}{\sqrt{2}}\left(\bar{\varepsilon} \gamma_{m} \gamma_{5} \lambda\right) \\
\gamma_{L} \delta_{b} \eta_{m} & =-\frac{1}{2} A_{m n}^{-}\left(\gamma^{n} \gamma_{R} \varepsilon\right)-\sqrt{2} \mathcal{D}_{m} \phi^{*}\left(\gamma_{L} \varepsilon\right)-\mu^{2} \gamma_{m}\left(\gamma_{R} \eta\right) \\
\gamma_{L} \delta_{b} \psi_{m} & =\frac{1}{2} A_{m n}^{-}\left(\gamma^{n} \gamma_{R} \eta\right)+\sqrt{2} \mathcal{D}_{m} \phi\left(\gamma_{L} \eta\right) \\
\delta_{b} e_{m}{ }^{a} & =0
\end{aligned}
$$

\section{The Single Antibrane}

Before moving on to a multi-brane example, we first pause to ask how the above construction changes if it is performed for a single antibrane rather than a single brane.

\subsection{The Brane - Antibrane Map}

Rather than performing the construction again from scratch, we instead directly write down the result based on the following two observations.

- First, if a brane breaks exactly half of the supersymmetries of the bulk space of a theory, then typically its antibrane leaves these supersymmetries unbroken, but breaks the other half of the supersymmetries.

- Second, in explicit microscopic constructions an antibrane configuration may often be simply obtained from a brane construction by performing a parity transformation on the brane world-volume.

There are several ways to see the origin of the world-volume parity transformation. For instance, in four spacetime dimensions the simplest field theory realization of a brane-like defect might be the vortex configuration of the abelian Higgs model, 
which is characterized by the nonzero magnetic flux which threads the vortex. The antivortex of this theory has the opposite magnetic flux, and is obtained simply by rotating the vortex $180^{\circ}$ about an axis perpendicular to the vortex. The effect of such a rotation is simply to reverse the spatial coordinate along the vortex. Alternatively, a similar argument applies if branes and antibranes are distinguished by how they couple to antisymmetric tensors, $B_{m_{1}, m_{2}, \ldots}$, which generically are proportional to $\int B$ over the brane's world volume. The result follows because this coupling changes sign if we reverse the brane's orientation - ie by a world-volume parity transformation.

These arguments indicate that the antibrane and brane actions should be obtainable from one another by performing a combination of a $4 \mathrm{D}$ parity transformation and an interchange of the roles of the two supersymmetries.

The same conclusion may also be drawn directly from the point of view of the $4 \mathrm{D} N=2$ supersymmetry algebra. In this algebra, the quantity which distinguishes a brane from an antibrane is the algebra's central charge, $Z$. This enters the $4 \mathrm{D}$ $N=2$ commutation relation through

$$
\left\{Q_{i}, \bar{Q}_{j}\right\}=-2 i \gamma_{m} P^{m} \delta_{i j}+i \gamma_{5} Z \epsilon_{i j}
$$

where $i, j=1,2$ labels the two supersymmetries, and $\epsilon_{i j}$ is the completely antisymmetric tensor. From eq. (3.1) it is clear that the effect of a parity transformation on the supersymmetry algebra is precisely the same as the effect of reversing the sign of the central charge.

Notice that the combined operation of parity plus an interchange of the two supersymmetries leaves the algebra, eq. (3.1), unchanged. It is straightforward to check that it also does not change the bulk part of the action and supersymmetry transformations, as these are defined in the previous section. The same is not true for the brane parts of the action or supersymmetry transformations, due to the property that the brane action realizes one of the supersymmetries linearly and the other nonlinearly.

A good first start is therefore to define the antibrane action and supersymmetry transformations by performing a parity transformation and supersymmetry interchange to the corresponding quantities for the brane. Actually, these two transformations alone would imply that the complex scalar, $\phi$, would become the supersymmetry partner of the right-handed (rather than the usual left-handed) fermion, so it is also convenient to replace $\phi \rightarrow \phi^{*}$ and $\chi \rightarrow-\chi$ when passing from brane to antibrane. This ensures that the matter transformations remain in their standard form. As is straightforward to check, the antibrane action which results from these replacements is automatically invariant under the antibrane supersymmetry transformation rules which result.

The pure bulk part of the action and supersymmetry transformations are unchanged by the combined action of parity and supersymmetry interchange, and so are given by eqs. (2.32) and (2.33). The antibrane action and transformations are 
directly obtained by performing a parity transformation and interchanging the fields as discussed above. Keeping in mind that parity changes the sign of $\gamma_{5}$ and $\epsilon^{m n p q}$, and interchanges $\gamma_{L} \leftrightarrow \gamma_{R}$ and $A_{m n}^{ \pm} \leftrightarrow A_{m n}^{\mp}$, we get in this way:

$$
\begin{aligned}
\frac{\mathcal{L}_{\tilde{b}}}{e}= & -\frac{1}{4} A_{m n} A^{m n}-\frac{1}{2} \bar{\chi} \not \chi \chi-\frac{1}{2} \bar{\lambda} \not \partial \lambda-\tilde{\mathcal{D}}_{m} \phi^{*} \tilde{\mathcal{D}}^{m} \phi-\frac{m}{2}\left(\bar{\psi}_{m} \gamma^{m n} \psi_{n}\right) \\
& -\frac{m}{2}(\bar{\chi} \chi)+i \sqrt{2} m\left(\bar{\lambda} \gamma_{5} \chi\right)+i m\left(\bar{\psi}_{m} \gamma_{5} \gamma^{m} \chi\right)-\frac{m}{\sqrt{2}}\left(\bar{\psi}_{m} \gamma^{m} \lambda\right) . \\
& +\frac{i \kappa}{\sqrt{2}}\left(\bar{\eta}_{m} \gamma^{n} \gamma^{m} \gamma_{L} \chi\right) \tilde{\mathcal{D}}_{n} \phi^{*}+\frac{\kappa}{2} \mathcal{A}_{-}^{m n}\left(\bar{\eta}_{m} \gamma_{n} \gamma_{R} \lambda\right) \\
& +\frac{i \kappa}{\sqrt{2} e} \epsilon^{m n p q}\left(\bar{\eta}_{m} \gamma_{n} \gamma_{R} \psi_{p}\right) \tilde{\mathcal{D}}_{q} \phi^{*}+\frac{\kappa}{2}\left(\bar{\eta}_{m} \gamma_{L} \psi_{n}\right) A_{+}^{m n}+\text { c.c.. }
\end{aligned}
$$

where (because of the change $\phi \rightarrow \phi^{*}$ ) we have for antibranes

$$
\tilde{\mathcal{D}}_{m} \phi=\partial_{m} \phi-m \mathcal{A}_{m}^{*}=\partial_{m} \phi-\frac{m}{\sqrt{2}}\left(A_{m}-i B_{m}\right) .
$$

The antibrane part of the supersymmetry transformations similarly becomes

$$
\begin{aligned}
\delta_{b} \phi & =i \sqrt{2} \bar{\eta} \gamma_{L} \chi \\
\gamma_{L} \delta_{b} \chi & =-i \sqrt{2} \tilde{\mathcal{D}}_{m} \phi\left(\gamma^{m} \gamma_{R} \eta\right)-2 i \mu^{2}\left(\gamma_{L} \varepsilon\right) \\
\gamma_{L} \delta_{b} \lambda & =-\frac{1}{2} \mathcal{A}_{m n}\left(\gamma^{m n} \gamma_{L} \eta\right)+\sqrt{2} \mu^{2}\left(\gamma_{L} \varepsilon\right) \\
\delta_{b} A_{m} & =\frac{1}{\sqrt{2}}\left(\bar{\eta} \gamma_{m} \lambda\right)-\left(\bar{\eta}_{m} \varepsilon-\bar{\psi}_{m} \eta\right) \\
\delta_{b} B_{m} & =-\frac{i}{\sqrt{2}}\left(\bar{\eta} \gamma_{m} \gamma_{5} \lambda\right) \\
\gamma_{L} \delta_{b} \eta_{m} & =+\frac{1}{2} A_{m n}^{-}\left(\gamma^{n} \gamma_{R} \varepsilon\right)+\sqrt{2} \tilde{\mathcal{D}}_{m} \phi\left(\gamma_{L} \varepsilon\right) \\
\gamma_{L} \delta_{b} \psi_{m} & =-\frac{1}{2} A_{m n}^{-}\left(\gamma^{n} \gamma_{R} \eta\right)-\sqrt{2} \tilde{\mathcal{D}}_{m} \phi^{*}\left(\gamma_{L} \eta\right)-\mu^{2}\left(\gamma_{m} \gamma_{R} \varepsilon\right) \\
\delta_{b} e_{m}{ }^{a} & =0
\end{aligned}
$$

\section{Two Branes}

Next consider how the above construction changes when there is more than one brane. We imagine that both of the branes break the same supersymmetry, and leave the same supersymmetry unbroken. What we expect to happen in this case would be that the bulk gravitino which is broken acquires its mass by mixing with a linear combination of the would-be Goldstone fermions on the two branes. The examination of this system allows us to see the extent to which the two branes can remain decoupled - or sequestered - from one another.

The minimal low-energy theory of the two brane configuration may be constructed by an unHiggsing process, much as was done for the single brane. The 
reason for this can be seen by counting the particle content for the two-brane system. As can be seen from the one brane system, each brane contributes at least a 4D $N=1$ chiral multiplet, $(\phi, \chi)$, and a gauge multiplet, $\left(\lambda, A_{m}\right)$. For two branes the low-energy theory contains two of each of these multiplets. The additional fields have precisely the content to fill out a single massive $N=1$ spin-1 supermultiplet, which has the particle content $\left(1, \frac{1}{2}, \frac{1}{2}, 0\right)$. The massive spin-3/2 field and the three massive vector fields therefore combine with the scalar and spin- $1 / 2$ fields into a massive $N=1$ spin-3/2 multiplet and a massive $N=1$ spin-1 multiplet.

Although the physical spectrum for the two-brane configuration is a massless $N=1$ spin $\left(2, \frac{3}{2}\right)$ multiplet, a massive $N=1$ spin $\left(\frac{3}{2}, 1,1, \frac{1}{2}\right)$ multiplet, and a massive $N=1$ spin $\left(1, \frac{1}{2}, \frac{1}{2}, 0\right)$ multiplet, we must still identify which linear combination of brane and bulk fields combines to form the mass eigenstates. We may do so by unHiggsing the two massive multiplets as was done above for the one-brane case.

\subsection{The Massive Spin-1 Multiplet}

To proceed we pause here to record the lagrangian density and global supersymmetry transformations for the massive $N=1$ spin-1 multiplet. We can then unHiggs it, and rotate to a 'brane' basis, in which the lagrangian and transformation rules look as much as possible like the sum of terms from two identical branes.

Consider therefore a massive $N=1$ multiplet with spin content $\left(1, \frac{1}{2}, \frac{1}{2}, 0\right)$, with the corresponding fields denoted by $\left\{A_{m}, \alpha, \beta, \varphi\right\}$. If the particle masses are denoted by $v$, then the free lagrangian density is:

$$
\begin{gathered}
\mathcal{L}_{v}=-\frac{1}{4} A_{m n} A^{m n}-\frac{1}{2} \bar{\alpha} \not \partial \alpha-\frac{1}{2} \bar{\beta} \not \partial \beta-\frac{1}{2} \partial_{m} \varphi \partial^{m} \varphi \\
-\frac{v^{2}}{2} A_{m} A^{m}-\frac{v}{2}(\bar{\alpha} \alpha+\bar{\beta} \beta)-\frac{v^{2}}{2} \varphi^{2} .
\end{gathered}
$$

As is shown in Appendix B, this action is invariant with respect to the global supersymmetry transformations:

$$
\begin{aligned}
\delta \varphi & =\frac{1}{\sqrt{2}} \bar{\varepsilon} \gamma_{L}(\beta+i \alpha)+c . c . \\
\gamma_{L} \delta \beta & =\frac{1}{\sqrt{2}}\left(\gamma^{m} \gamma_{R} \varepsilon\right)\left[\partial_{m} \varphi+i v A_{m}\right]-\frac{v}{\sqrt{2}} \varphi\left(\gamma_{L} \varepsilon\right)+\frac{i}{2 \sqrt{2}} A_{m n}\left(\gamma^{m n} \gamma_{L} \varepsilon\right) \\
\gamma_{L} \delta \alpha & =-\frac{i}{\sqrt{2}}\left(\gamma^{m} \gamma_{R} \varepsilon\right)\left[\partial_{m} \varphi+i v A_{m}\right] \\
& -\frac{i v}{\sqrt{2}} \varphi\left(\gamma_{L} \varepsilon\right)-\frac{1}{2 \sqrt{2}} A_{m n}\left(\gamma^{m n} \gamma_{L} \varepsilon\right) \\
\delta A_{m} & =-\partial_{m}\left[\frac{i}{v \sqrt{2}} \bar{\varepsilon} \gamma_{L}(\beta+i \alpha)\right]+\frac{i}{\sqrt{2}} \bar{\varepsilon} \gamma_{m} \gamma_{L}(\beta-i \alpha)+c . c .
\end{aligned}
$$

It also shows convenient to define the new (canonically normalized Majorana) fermions $\chi=\left(\beta+i \gamma_{5} \alpha\right) / \sqrt{2}$ and $\lambda=\left(\beta-i \gamma_{5} \alpha\right) / \sqrt{2}$, which have more conventional 
transformation rules. With these choices we have:

$$
\begin{aligned}
\delta \varphi & =\bar{\varepsilon} \gamma_{L} \chi+c . c . \\
\gamma_{L} \delta \chi & =\left(\gamma^{m} \gamma_{R} \varepsilon\right)\left[\partial_{m} \varphi+i v A_{m}\right] \\
\gamma_{L} \delta \lambda & =-v \varphi\left(\gamma_{L} \varepsilon\right)+\frac{i}{2} A_{m n}\left(\gamma^{m n} \gamma_{L} \varepsilon\right) \\
\delta A_{m} & =-\partial_{m}\left[\frac{i}{v}\left(\bar{\varepsilon} \gamma_{L} \chi\right)\right]+i\left(\bar{\varepsilon} \gamma_{m} \gamma_{L} \lambda\right)+\text { c.c. }
\end{aligned}
$$

This result may be unHiggsed by shifting $A_{m}^{\text {old }}=A_{m}^{\text {new }}-\frac{1}{v} \partial_{m} \sigma$, and requiring all $1 / v$ terms to vanish as a result. We find the unHiggsed lagrangian is

$$
\begin{gathered}
\mathcal{L}_{v}=-\frac{1}{4} A_{m n} A^{m n}-\frac{1}{2} \bar{\lambda} \not \partial \lambda-\frac{1}{2} \bar{\chi} \not \chi-\frac{1}{2} \partial_{m} \varphi \partial^{m} \varphi \\
-\frac{1}{2} \mathcal{D}_{m} \sigma \mathcal{D}^{m} \sigma-v(\bar{\chi} \lambda)-\frac{v^{2}}{2} \varphi^{2},
\end{gathered}
$$

where $\mathcal{D}_{m} \sigma=\partial_{m} \sigma-v A_{m}$.

Writing $\phi=(\sigma+i \varphi) / \sqrt{2}$ we have the supersymmetry transformations

$$
\begin{aligned}
\delta \phi & =i \sqrt{2}\left(\bar{\varepsilon} \gamma_{L} \chi\right) \\
\gamma_{L} \delta \chi & =-i \sqrt{2}\left(\gamma^{m} \gamma_{R} \varepsilon\right)\left[\partial_{m} \phi-\frac{v}{\sqrt{2}} A_{m}\right] \\
\gamma_{L} \delta \lambda & =\frac{i v}{\sqrt{2}}\left(\phi-\phi^{*}\right)\left(\gamma_{L} \varepsilon\right)+\frac{i}{2} A_{m n}\left(\gamma^{m n} \gamma_{L} \varepsilon\right) \\
\delta A_{m} & =i\left(\bar{\varepsilon} \gamma_{m} \gamma_{L} \lambda\right)+\text { c.c. }=i \bar{\varepsilon} \gamma_{m} \gamma_{5} \lambda
\end{aligned}
$$

These transformation rules suggest the definition $\mathcal{D}_{m} \phi=\partial_{m} \phi-\frac{v}{\sqrt{2}} A_{m}$.

\subsection{The Two-Brane System}

We now couple the spin-3/2 and spin- 1 supermultiplets to the massless spin- 2 multiplet of unbroken $N=1$ supergravity. We imagine that the branes are identical, and so contribute equally to the breaking of the broken supersymmetry.

To this end, we take the bulk matter content to be $\left\{e^{a}{ }_{m}, \psi_{m}, \eta_{m}, B_{m}\right\}$, and supplement this with two copies of the brane matter: $\left\{A_{m}, \lambda, \chi, \phi\right\}$ and $\left\{A_{m}^{\prime}, \lambda^{\prime}, \chi^{\prime}, \phi^{\prime}\right\}$. In general these fields combine into a massive spin- $3 / 2$ multiplet plus a massive spin-1 multiplet, whose masses need not be equal. We consider the case where both multiplets have the same mass, which we denote by $m$.

Under these assumptions the brane lagrangian is given by the following expression when written in terms of mass eigenstates: $\mathcal{L}=\mathcal{L}_{B}+\mathcal{L}_{2 b}$, where $\mathcal{L}_{B}$ is the bulk lagrangian given earlier, eq. (2.32). The brane part of the action is the sum of the massive spin-3/2 and spin-1 lagrangians: $\mathcal{L}_{2 b}=\mathcal{L}_{3 / 2}+\mathcal{L}_{1}$, where

$$
\frac{\mathcal{L}_{3 / 2}}{e}=-\frac{1}{4} A_{m n} A^{m n}-\frac{1}{2} \bar{\chi} \not \partial \chi-\frac{1}{2} \bar{\lambda} \not \partial \lambda-\mathcal{D}_{m} \phi^{*} \mathcal{D}^{m} \phi-\frac{m}{2}\left(\bar{\eta}_{m} \gamma^{m n} \eta_{n}\right)
$$




$$
-\frac{m}{2}(\bar{\chi} \chi)+i \sqrt{2} m\left(\bar{\lambda} \gamma_{5} \chi\right)+i m\left(\bar{\eta}_{m} \gamma_{5} \gamma^{m} \chi\right)-\frac{m}{\sqrt{2}}\left(\bar{\eta}_{m} \gamma^{m} \lambda\right)
$$

and

$$
\begin{gathered}
\mathcal{L}_{1}=-\frac{1}{4} A_{m n}^{\prime} A^{\prime m n}-\frac{1}{2} \bar{\lambda}^{\prime} \not \partial \lambda^{\prime}-\frac{1}{2} \bar{\chi}^{\prime} \not \partial \chi^{\prime}-\mathcal{D}_{m} \phi^{\prime *} \mathcal{D}^{m} \phi^{\prime} \\
-m\left(\bar{\chi}^{\prime} \lambda^{\prime}\right)+\frac{m^{2}}{4}\left(\phi^{\prime}-\phi^{\prime *}\right)^{2}
\end{gathered}
$$

where

$$
\mathcal{D}_{m} \phi=\partial_{m} \phi-\frac{m}{\sqrt{2}}\left(A_{m}+i B_{m}\right), \quad \mathcal{D}_{m} \phi^{\prime}=\partial_{m} \phi^{\prime}-\frac{m}{\sqrt{2}} A_{m}^{\prime} .
$$

Both $\mathcal{L}_{3 / 2}$ and $\mathcal{L}_{1}$ have the supersymmetries we have worked out, with the fields in $\mathcal{L}_{3 / 2}$ transforming as in eqs. (2.35) and those in $\mathcal{L}_{1}$ transforming as in eqs. (4.5). We wish to now rotate the matter multiplets to a brane basis, for which the massless limit is nonsingular and the two brane contributions look similar to one another. Since we assume both branes contribute equally to the breaking of the supersymmetry, we can assume a symmetry under their interchange, and so we can assume that the required rotation between the brane states and the mass eigenstates is through 45 degrees. We therefore take:

$$
\begin{array}{cc}
\left(\begin{array}{c}
A_{m} \\
A_{m}^{\prime}
\end{array}\right)=R\left(\begin{array}{c}
A_{m}^{1} \\
A_{m}^{2}
\end{array}\right), \quad\left(\begin{array}{c}
\lambda \\
\lambda^{\prime}
\end{array}\right)=R\left(\begin{array}{l}
\lambda_{1} \\
\lambda_{2}
\end{array}\right), \\
\left(\begin{array}{c}
\chi \\
\chi^{\prime}
\end{array}\right)=R\left(\begin{array}{l}
\chi_{1} \\
\chi_{2}
\end{array}\right), \quad\left(\begin{array}{c}
\phi \\
\phi^{\prime}
\end{array}\right)=R\left(\begin{array}{l}
\phi_{1} \\
\phi_{2}
\end{array}\right),
\end{array}
$$

with

$$
R=\left(\begin{array}{cc}
c_{\theta} & s_{\theta} \\
-s_{\theta} & c_{\theta}
\end{array}\right)=\frac{1}{\sqrt{2}}\left(\begin{array}{cc}
1 & 1 \\
-1 & 1
\end{array}\right)
$$

where $c_{\theta}=\cos \theta$ and $s_{\theta}=\sin \theta$.

Notice if we define:

$$
\mathcal{D}_{m} \phi_{1}=\partial_{m} \phi_{1}-\frac{m}{\sqrt{2}}\left(A_{m}^{1}+\frac{i}{\sqrt{2}} B_{m}\right), \quad \mathcal{D}_{m} \phi_{2}=\partial_{m} \phi_{2}-\frac{m}{\sqrt{2}}\left(A_{m}^{2}+\frac{i}{\sqrt{2}} B_{m}\right)
$$

then we also have:

$$
\left(\begin{array}{c}
\mathcal{D}_{m} \phi \\
\mathcal{D}_{m} \phi^{\prime}
\end{array}\right)=R\left(\begin{array}{c}
\mathcal{D}_{m} \phi_{1} \\
\mathcal{D}_{m} \phi_{2}
\end{array}\right)
$$

The brane action is obtained by inserting this rotation into the above lagrangian, and the supersymmetries are similarly found by using this rotation in the known supersymmetry transformations. In this way we find $\mathcal{L}_{2 b}=\mathcal{L}_{b 1}+\mathcal{L}_{b 2}+\mathcal{L}_{b 1 b 2}$ with:

$$
\begin{aligned}
\mathcal{L}_{b 1}=-\frac{1}{4} & A_{m n}^{1} A_{1}^{m n}-\frac{1}{2} \bar{\lambda}_{1} \not \partial \lambda_{1}-\frac{1}{2} \bar{\chi}_{1} \not \partial \chi_{1}-\mathcal{D}_{m} \phi_{1}^{*} \mathcal{D}^{m} \phi_{1} \\
+ & \frac{i m}{\sqrt{2}}\left(\bar{\eta}_{m} \gamma_{5} \gamma^{m} \chi_{1}\right)-\frac{m}{2}\left(\bar{\eta}_{m} \gamma^{m} \lambda_{1}\right)-\frac{m}{4}\left(\bar{\eta}_{m} \gamma^{m n} \eta_{n}\right) \\
+ & \frac{m^{2}}{8}\left(\phi_{1}-\phi_{1}^{*}\right)^{2}-\frac{m}{4}\left[\left(\bar{\chi}_{1} \chi_{1}\right)+2 \bar{\chi}_{1}\left(1-i \sqrt{2} \gamma_{5}\right) \lambda_{1}\right]
\end{aligned}
$$


and $\mathcal{L}_{b 2}$ obtained from this by replacing $1 \rightarrow 2$ everywhere. The direct brane-brane mixing terms are:

$$
\begin{aligned}
\mathcal{L}_{b 1 b 2}=-\frac{m^{2}}{4} & \left(\phi_{1}-\phi_{1}^{*}\right)\left(\phi_{2}-\phi_{2}^{*}\right) \\
& -\frac{m}{2}\left[\left(\bar{\chi}_{1} \chi_{2}\right)-\bar{\chi}_{1}\left(1+i \sqrt{2} \gamma_{5}\right) \lambda_{2}-\bar{\chi}_{2}\left(1+i \sqrt{2} \gamma_{5}\right) \lambda_{1}\right] .
\end{aligned}
$$

The supersymmetry transformation laws are similarly obtained by substitution of the field rotation into those rules which were previously derived. One finds the brane-field-dependent part of the bulk-field transformation laws to be

$$
\begin{aligned}
\delta_{b} B_{m}= & \frac{i}{2} \bar{\varepsilon} \gamma_{m} \gamma_{5}\left(\lambda_{1}+\lambda_{2}\right) \\
\gamma_{L} \delta_{b} \eta_{m}= & -\frac{1}{2 \sqrt{2}}\left(A_{m n}^{1-}+A_{m n}^{2-}\right)\left(\gamma^{n} \gamma_{R} \varepsilon\right) \\
& \quad-\left(\mathcal{D}_{m} \phi_{1}^{*}+\mathcal{D}_{m} \phi_{2}^{*}\right)\left(\gamma_{L} \varepsilon\right)-\frac{m}{\kappa} \gamma_{m}\left(\gamma_{R} \eta\right) \\
\gamma_{L} \delta_{b} \psi_{m}= & \frac{1}{2 \sqrt{2}}\left(A_{m n}^{1-}+A_{m n}^{2-}\right)\left(\gamma^{n} \gamma_{R} \eta\right)+\left(\mathcal{D}_{m} \phi_{1}+\mathcal{D}_{m} \phi_{2}\right)\left(\gamma_{L} \eta\right) \\
\delta_{b} e_{m}{ }^{a}= & 0 .
\end{aligned}
$$

The transformations of the brane fields become:

$$
\begin{aligned}
& \delta \phi_{1}=i \sqrt{2} \bar{\varepsilon} \gamma_{L} \chi_{1} \\
& \gamma_{L} \delta \chi_{1}=-i \sqrt{2} \mathcal{D}_{m} \phi_{1}\left(\gamma^{m} \gamma_{R} \varepsilon\right)-i \sqrt{2} \frac{m}{\kappa}\left(\gamma_{L} \eta\right) \\
& \gamma_{L} \delta \lambda_{1}=-\frac{1}{4 \sqrt{2}}\left[(1-i \sqrt{2}) A_{m n}^{1}+(1+i \sqrt{2}) A_{m n}^{2}-i \sqrt{2} B_{m n}\right]\left(\gamma^{m n} \gamma_{L} \varepsilon\right)+\frac{i m}{2 \sqrt{2}}\left(\phi_{1}-\phi_{2}-\phi_{1}^{*}+\phi_{2}^{*}\right)\left(\gamma_{L} \varepsilon\right)+\frac{m}{\kappa}\left(\gamma_{L} \eta\right) \\
& \delta A_{m}^{1}=\frac{1}{2 \sqrt{2}} \bar{\varepsilon} \gamma_{m}\left(1+i \sqrt{2} \gamma_{5}\right) \lambda_{1}+\frac{1}{2 \sqrt{2}} \bar{\varepsilon} \gamma_{m}\left(1-i \sqrt{2} \gamma_{5}\right) \lambda_{2} \\
&+\frac{1}{\sqrt{2}}\left(\bar{\eta}_{m} \varepsilon-\bar{\psi}_{m} \eta\right) .
\end{aligned}
$$

with the transformation rule for brane- 2 fields obtained by making the substitutions $1 \leftrightarrow 2$ in these expressions.

\subsection{Noether Coupling to Two Branes}

The couplings to supergravity which arise from the Noether prescription are again easily identified. Starting with the massive spin-1 multiplet, eq. (4.7), inserting covariant derivatives throughout and following the derivatives of the supersymmetry parameter in the variation of the action, one finds:

$$
\begin{gathered}
\frac{\mathcal{L}_{1 \kappa}}{e}=\frac{i \kappa m}{2 \sqrt{2}} \bar{\psi}_{m} \gamma^{m} \gamma_{L} \lambda^{\prime}\left(\phi^{\prime}-\phi^{\prime *}\right)+\frac{i \kappa}{2} \bar{\psi}_{m} \gamma_{n} \gamma_{L} \lambda^{\prime} A_{+}^{\prime m n} \\
+\frac{i \kappa}{\sqrt{2}} \bar{\psi}_{m} \gamma^{n} \gamma^{m} \gamma_{L} \chi^{\prime} \mathcal{D}_{n} \phi^{\prime *}+\text { c.c. }
\end{gathered}
$$


This, when combined with eq. (2.27), gives the complete $\mathcal{O}(\kappa)$ Noether couplings for the two brane system.

Substitution of the rotations of eq. (4.9) into these expressions gives them in terms of the brane fields $\phi_{1}, \phi_{2}$ etc.. Once this is done it is clear that terms arise at $\mathcal{O}(\kappa)$ which contribute to the direct couplings between fields arising on each brane.

\section{Conclusions}

We have studied the form of the low-energy action for brane configurations which partially break $4 \mathrm{D} N=2$ supersymmetry down to $4 \mathrm{D} N=1$ supersymmetry. Our focus has been on those fields which are necessarily present in this kind of symmetry breaking pattern, because they are required in order to fill out the required $N=$ 1 supersymmetry multiplets which contain the massive spin-3/2 and spin- 1 fields which arise. We have seen how the linear realization of one supersymmetry and the nonlinear realization of the other strongly constrain the form the resulting low-energy action can take.

In particular, our analysis allows us to address the sequestering conjecture of ref. [7], in which a strong decoupling of the degrees of freedom on two supersymmetric branes is proposed. We can test a part of the sequestered form for the Kähler potential, eq. (1.1), since this implies that the terms which directly mix the chiral multiplets of the two branes arise for the first time at $\mathcal{O}\left(\kappa^{2}\right)$ in the effective lagrangian.

We find that this kind of sequestered decoupling is not satisfied by the degrees of freedom we follow in the low-energy theory, since direct brane-brane contact couplings arise already at $\mathcal{O}(\kappa)$, as may be seen from eq. (4.14) if we use the natural relation $m=\kappa \mu^{2}$ to see that $m$ itself is $\mathcal{O}(\kappa)$ relative to the size, $\mu^{2}$, of the supersymmetry-breaking order parameter. A similar conclusion follows from an inspection of the Noether couplings for two branes.

These findings complement and reinforce those of ref. [9], who examined the couplings between branes in specific types of string theory compactifications for which direct brane-brane couplings are explicitly calculable and attributable to exchanges of bulk supergravity fields, or to the warping of the internal compact dimensions. The present analysis shows that these contact terms do not depend on the details of these compactifications, but follow quite robustly from the assumed 4D pattern of partial supersymmetry breaking.

The phenomenological appeal of low-energy sequestering for circumventing the flavor problems of low-energy supersymmetry breaking makes the continued search for models with sequestered sectors well worthwhile. Our results show that any such system cannot be obtained simply by coupling additional fields which do not participate in the diagonalization of the gravitino masses. Instead a more radical change is required, and it may be that what is required is a supersymmetry-breaking 
pattern within which a larger extended supersymmetry is partially broken, such as perhaps $N=4$ breaking to $N=2$. Some evidence that this might be the case can be found from the expression of these extended supersymmetries in terms of $N=1$ superfields [20], which are often suggestive of sequestering.

Our techniques can clearly be extended to the complete breaking of $N=2$ supersymmetry, such as is obtained by combining the supersymmetry breaking of one brane with that of an antibrane. In such a system we naturally expect that the full $N=2$ supersymmetry will be nonlinearly realized, and the challenge is to find what additional constraints distinguish the resulting low-energy theory from a generic nonsupersymmetric model. We see the present work as providing the first step towards this more ambitious project of studying the low-energy implications of supersymmetry breaking in the brane world. This is of particular relevance given the fact that there are explicit constructions of D-brane models for which either supersymmetry is broken by brane/antibrane systems, or in intersecting brane models where different intersecting branes break different supersymmetries, whereas the bulk preserves higher supersymmetries. This kind of 'non-local' realization of supersymmetry breaking by many branes in a supersymmetric bulk was proposed in [6] as a way to ameliorate the cosmological constant problem, and has been recently constructed in intersecting D-brane models [21], where it was called quasi-supersymmetry (QSUSY). It is clearly of great interest to study the low-energy limits of these models and uncover whether the brane constructions lead to new low-energy mechanisms for keeping parameters naturally small. We hope to report on some of these issues in future publications.

\section{Acknowledgments}

We thank L. Ibáñez for interesting conversations. C.B.'s research is partially funded by grants from N.S.E.R.C. of Canada and F.C.A.R. of Québec. C.B. and F.Q. respectively thank D.A.M.T.P. of Cambridge University and the CERN Theory Division for their hospitality during various parts of this work. M.K. wants to thank R. Altendorfer for useful e-mail correspondence. The work of M.K. is currently funded by the Deutsche Forschungsgemeinschaft, but the major part of this work was performed while M.K. was an SPG fellow at the University of Cambridge supported by PPARC. F.Q. is partially supported by PPARC.

\section{A. Conventions}

Since supersymmetric calculations tend to be finicky, and since conventions vary across the supersymmetry literature, we spell out our conventions in detail in this appendix. 


\section{A.1 Majorana spinor formalism}

We use Majorana spinors throughout, with 4-component gamma matrices. The notation closely follows that of Weinberg's textbook [18 — with the exception of the sign we use for the gravitino mass term - and is similar to that used in West's book [22]. In particular we use Weinberg's curvature conventions, which differ by an overall minus sign from the curvature conventions of West and of Misner, Thorne and Wheeler [23].

\section{Basic Definitions}

$$
\begin{array}{cc}
\text { metric: } \quad \eta^{m n}=\operatorname{diag}(-+++) & \epsilon^{0123}=-\epsilon_{0123}=1 \\
\text { epsilon tensor: } & \epsilon_{m n p q} \epsilon^{m a b c}=-\left(\delta_{n}^{a} \delta_{p}^{b} \delta_{q}^{c} \pm 5 \text { perms }\right) \\
\epsilon_{m n p q} \epsilon^{m n a b}=-2\left(\delta_{p}^{a} \delta_{q}^{b}-\delta_{q}^{a} \delta_{p}^{b}\right) & \tilde{t}_{m n}=\frac{i}{2} \epsilon_{m n a b} t^{a b} \\
\text { Dual Tensors: }
\end{array}
$$

(Anti) Selfdual Tensors: $\quad t_{ \pm}^{m n}=t^{m n} \pm \frac{i}{2} \epsilon^{m n a b} t_{a b}=t^{m n} \pm \tilde{t}^{m n} \quad \tilde{t}_{ \pm}^{m n}= \pm t_{ \pm}^{m n}$

Dirac matrices: $\quad\left\{\gamma^{m}, \gamma^{n}\right\}=2 \eta^{m n}$

$$
\text { Commutator: } \quad \gamma^{m n}=\frac{1}{2}\left[\gamma^{m}, \gamma^{n}\right]
$$

$$
\text { Projectors: } \quad \gamma_{L}=\frac{1}{2}\left(1+\gamma_{5}\right), \quad \gamma_{R}=\frac{1}{2}\left(1-\gamma_{5}\right)
$$

\section{Flat-Space Dirac Identities}

$$
\begin{gathered}
\gamma_{m} \gamma_{n}=\eta_{m n}+\gamma_{m n} \\
\gamma_{5} \gamma_{m n}=-\frac{i}{2} \epsilon_{m n p q} \gamma^{p q} \\
\epsilon^{m n p q} \gamma_{5} \gamma_{n a}=-i\left(\delta_{a}^{m} \gamma^{p q}-\delta_{a}^{p} \gamma^{m q}+\delta_{a}^{q} \gamma^{m p}\right) \\
\epsilon^{m n a b} \gamma_{5} \gamma_{a b}=2 i \gamma^{m n} \\
\gamma_{m} \gamma_{n p}=\left(\eta_{m n} \gamma_{p}-\eta_{m p} \gamma_{n}\right)+i \epsilon_{m n p q} \gamma_{5} \gamma^{q} \\
\gamma_{n p} \gamma_{m}=-\left(\eta_{m n} \gamma_{p}-\eta_{m p} \gamma_{n}\right)+i \epsilon_{m n p q} \gamma_{5} \gamma^{q} \\
\gamma_{m} \gamma_{n} \gamma_{p}=\gamma_{m} \eta_{n p}+\gamma_{p} \eta_{m n}-\gamma_{n} \eta_{m p}+i \epsilon_{m n p q} \gamma_{5} \gamma^{q} \\
{\left[\gamma_{m n}, \gamma_{n p}-\eta_{m p} \eta_{n q}+i \gamma_{5} \epsilon_{m n p q}-\eta_{m p} \gamma_{n q}+\eta_{n p} \gamma_{m q}-\eta_{n q} \gamma_{m p}+\eta_{m q} \gamma_{n p}\right.} \\
\left\{\gamma_{m n}, \gamma_{n q}+\eta_{n p} \gamma_{m q}-\eta_{n q} \gamma_{m p}+\eta_{m q} \gamma_{n p}\right)
\end{gathered}
$$




\section{Lorentz Transformations}

Lorentz Generators: $\quad$ 4-vectors: $\quad \delta V_{m}=\frac{i}{2} \omega^{a b}\left(J_{a b}\right)_{m}{ }^{n} V_{n}=\omega_{m}^{n} V_{n}$

This implies for 4 vectors: $\quad\left(J_{a b}\right)_{m}{ }^{n}=-i\left(\eta_{a m} \delta_{b}^{n}-\eta_{b m} \delta_{a}^{n}\right)$

This fixes the Lorentz Commutators: $\quad\left[J_{a b}, J_{c d}\right]=-i \eta_{b c} J_{m d} \pm$ permutations

Spinor Lorentz generators: $\quad \delta \psi=\frac{i}{2} \omega^{a b} J_{a b} \psi, \quad J_{a b}=-\frac{i}{2} \gamma_{a b}$

Spinor Covariant Derivative: $\quad D_{m} \psi=\partial_{m} \psi+\frac{i}{2} \omega_{m}^{a b} J_{a b} \psi=\partial_{m} \psi+\frac{1}{4} \omega_{m}{ }^{a b} \gamma_{a b} \psi$

Curvature Commutator - spinor: $\quad\left[D_{m}, D_{n}\right] \psi=-\frac{i}{2} R_{m n}{ }^{a b} J_{a b} \psi=-\frac{1}{4} R_{m n}{ }^{a b} \gamma_{a b} \psi$

Curvature Commutator - vector: $\quad\left[D_{m}, D_{n}\right] V_{p}=-\frac{i}{2} R_{m n}{ }^{a b}\left(J_{a b}\right)_{p}{ }^{q} V_{q}=R_{m n p}{ }^{q} V_{q}$

Majorana Spinors If $\chi$ and $\psi$ are any Majorana spinors, then:

Reality: $\quad(\bar{\chi} M \psi)^{*}= \pm \bar{\chi} M \psi ; \quad$ with + for $M=S, V, T$ and - for $M=P, A$

Symmetry: $\quad \bar{\chi} M \psi= \pm \bar{\psi} M \chi ; \quad$ with + for $M=S, P, A$ and - for $M=V, T$

\section{A.2 Weyl spinor formalism}

We would like to show how the results of this article can be translated into the Weyl spinor formalism of Wess and Bagger [24]. The Weyl spinor components of a Dirac 4-spinor $\Psi$ are defined through

$$
\psi \equiv \overline{\gamma_{L} \Psi}=\bar{\Psi} \gamma_{R}, \quad \chi \equiv \gamma_{R} \Psi
$$

If $\Psi$ is restricted to satisfy a Majorana condition, then $\chi=\psi$. In this case, $\Psi$ can be written as

$$
\Psi_{a}=\left(\begin{array}{c}
\bar{\psi}^{\dot{\alpha}} \\
\psi_{\alpha}
\end{array}\right) \quad \Rightarrow \quad \bar{\Psi}^{a}=\left(\bar{\psi}_{\dot{\alpha}}, \psi^{\alpha}\right)
$$

where $a=(\dot{\alpha}, \alpha)$ is a 4 -spinor index. Similarly, the $\gamma$-matrices can be split into blocks of $\sigma$-matrices:

$$
\left(\gamma^{m}\right)_{a}^{b}=i\left(\begin{array}{cc}
0 & \left(\bar{\sigma}^{m}\right)^{\dot{\alpha} \beta} \\
\left(\sigma^{m}\right)_{\alpha \dot{\beta}} & 0
\end{array}\right), \quad\left(\gamma^{m n}\right)_{a}^{b}=-2\left(\begin{array}{cc}
\left(\bar{\sigma}^{m n}\right)^{\dot{\alpha}} & 0 \\
0 & \left(\sigma^{m n}\right)_{\alpha}^{\beta}
\end{array}\right)
$$

To make contact with the notation of [14], we define two Weyl spinor gravitini $\psi^{(1)}, \psi^{(2)}$ through

$$
\eta_{m}=i \gamma_{5}\left(\begin{array}{c}
\bar{\psi}_{m}^{(2)} \\
\psi_{m}^{(2)}
\end{array}\right), \quad \psi_{m}=\left(\begin{array}{c}
\bar{\psi}_{m}^{(1)} \\
\psi_{m}^{(1)}
\end{array}\right)
$$


The chiral rotation of the second gravitino was necessary because we have chosen an unconventional sign for the gravitino mass term in our lagrangians. Similarly, we define the Weyl spinor supersymmetry transformation parameters $\eta^{(1)}, \eta^{(2)}$ through

$$
\varepsilon=\left(\begin{array}{l}
\bar{\eta}^{(1)} \\
\eta^{(1)}
\end{array}\right), \quad \eta=i \gamma_{5}\left(\begin{array}{l}
\bar{\eta}^{(2)} \\
\eta^{(2)}
\end{array}\right) .
$$

The Weyl spinor Goldstino $\nu$ is defined by

$$
\nu=-\gamma_{R} \xi
$$

In this section, we denote by $\zeta, \chi, \lambda$ the Weyl spinor components of the Majorana spinors $\zeta, \chi, \lambda$ in the main text, $i e$, we replace

$$
\gamma_{R} \zeta \rightarrow \zeta, \quad \gamma_{R} \chi \rightarrow \chi, \quad \gamma_{R} \lambda \rightarrow \lambda .
$$

Finally, we replace

$$
\mathcal{A}_{m} \rightarrow \frac{i}{\sqrt{2}} \overline{\mathcal{A}}_{m}, \quad \phi \rightarrow i \bar{\phi} .
$$

In this notation the unHiggsed massive gravitino lagrangian (2.9) reads

$$
\begin{aligned}
\mathcal{L}= & \epsilon^{m n p q} \bar{\psi}_{m}^{(2)} \bar{\sigma}_{n} \partial_{p} \psi_{q}^{(2)}-i \bar{\nu} \bar{\sigma}^{m} \partial_{m} \nu-i \bar{\zeta} \bar{\sigma}^{m} \partial_{m} \zeta-\mathcal{D}^{m} \phi \overline{\mathcal{D}}_{m} \bar{\phi}-\frac{1}{4} \mathcal{F}^{m n} \overline{\mathcal{F}}_{m n} \\
& -m\left(\psi_{m}^{(2)} \sigma^{m n} \psi_{n}^{(2)}+\frac{3 i}{\sqrt{6}} \psi_{m}^{(2)} \sigma^{m} \bar{\nu}+\nu \nu+\frac{1}{2} \zeta \zeta+\text { h.c. }\right)
\end{aligned}
$$

where $\mathcal{F}_{m n}=\partial_{[m} \mathcal{A}_{n]}$ and $\mathcal{D}_{m} \phi=\partial_{m} \phi-\frac{m}{\sqrt{2}} \mathcal{A}_{m}$.

The corresponding transformation laws are

$$
\begin{aligned}
\delta_{\eta} \psi_{m} & =-\frac{i}{2} \mathcal{F}_{+m n} \sigma^{n} \bar{\eta}+\sqrt{2} \overline{\mathcal{D}}_{m} \bar{\phi} \eta \\
\delta_{\eta} \nu & =-\frac{1}{\sqrt{6}}\left(\overline{\mathcal{F}}_{m n} \sigma^{m n} \eta-2 \sqrt{2} i \mathcal{D}_{n} \phi \sigma^{n} \bar{\eta}\right) \\
\delta_{\eta} \mathcal{A}_{m} & =2 \psi_{m} \eta-\frac{2 i}{\sqrt{6}}(-i \sqrt{2} \bar{\zeta}-\bar{\nu}) \bar{\sigma}_{m} \eta \\
\delta_{\eta} \phi & =\sqrt{\frac{2}{3}}(i \zeta+\sqrt{2} \nu) \eta \\
\delta_{\eta} \zeta & =-\frac{i}{\sqrt{3}} \overline{\mathcal{F}}_{m n} \sigma^{m n} \eta+\sqrt{\frac{2}{3}} \mathcal{D}_{m} \phi \sigma^{m} \bar{\eta}
\end{aligned}
$$

The Noether coupling of the massive gravitino lagrangian to supergravity yields

$$
\begin{aligned}
e^{-1} \mathcal{L}= & -\frac{1}{2 \kappa^{2}} R+\epsilon^{m n p q} \bar{\psi}_{m}^{(i)} \bar{\sigma}_{n} D_{p} \psi_{q}^{(i)}-i \bar{\chi} \bar{\sigma}^{m} D_{m} \chi-i \bar{\lambda} \bar{\sigma}^{m} D_{m} \lambda \\
& -\mathcal{D}^{m} \phi \overline{\mathcal{D}}_{m} \bar{\phi}-\frac{1}{4} \mathcal{F}^{m n} \overline{\mathcal{F}}_{m n}
\end{aligned}
$$




$$
\begin{aligned}
& -m\left(\frac{1}{\sqrt{2}} \psi_{m}^{(2)} \sigma^{m} \bar{\lambda}+i \psi_{m}^{(2)} \sigma^{m} \bar{\chi}+i \sqrt{2} \lambda \chi+\frac{1}{2} \chi \chi+\psi_{m}^{(2)} \sigma^{m n} \psi_{n}^{(2)}\right) \\
& -\frac{\kappa}{\sqrt{2}}\left(\chi \sigma^{m} \bar{\sigma}^{n} \psi_{m}^{(1)} \overline{\mathcal{D}}_{n} \bar{\phi}+\frac{1}{2} \bar{\lambda} \bar{\sigma}_{m} \psi_{n}^{(1)} \overline{\mathcal{F}}_{-}^{m n}+\epsilon^{m n p q} \bar{\psi}_{m}^{(2)} \bar{\sigma}_{n} \psi_{p}^{(1)} \overline{\mathcal{D}}_{q} \bar{\phi}\right) \\
& +\frac{\kappa}{4} \epsilon^{i j} \psi_{m}^{(i)} \psi_{n}^{(j)} \overline{\mathcal{F}}_{+}^{m n}+\text { h.c. },
\end{aligned}
$$

which is the Weyl spinor expression of the lagrangian (2.25), (2.27). Here, we defined

$$
\chi \equiv \frac{1}{\sqrt{3}}(i \zeta+\sqrt{2} \nu), \quad \lambda \equiv \frac{1}{\sqrt{3}}(-\sqrt{2} \zeta-i \nu) .
$$

The corresponding transformation laws (2.31) are

$$
\begin{aligned}
\delta_{\eta} e_{m}^{a}= & i \kappa\left(\eta^{(i)} \sigma^{a} \bar{\psi}_{m}^{(i)}+\bar{\eta}^{(i)} \bar{\sigma}^{a} \psi_{m}^{(i)}\right) \\
\delta_{\eta} \psi_{m}^{(i)}= & \frac{2}{\kappa} D_{m} \eta^{(i)}+\frac{i}{2} \epsilon^{i j} \mathcal{F}_{+m n} \sigma^{n} \bar{\eta}^{(j)}+\sqrt{2}\left(\overline{\mathcal{D}}_{m} \bar{\phi} \eta^{(1)} \delta^{i 2}-\mathcal{D}_{m} \phi \eta^{(2)} \delta^{i 1}\right) \\
& +i \mu^{2} \sigma_{m} \bar{\eta}^{(2)} \delta^{i 2} \\
\delta_{\eta} \mathcal{A}_{m}= & -2 \epsilon^{i j} \psi_{m}^{(i)} \eta^{(j)}+\sqrt{2} \bar{\lambda} \bar{\sigma}_{m} \eta^{(1)} \\
\delta_{\eta} \lambda= & \frac{i}{\sqrt{2}} \overline{\mathcal{F}}_{m n} \sigma^{m n} \eta^{(1)}-i \sqrt{2} \mu^{2} \eta^{(2)} \\
\delta_{\eta} \chi= & i \sqrt{2} \sigma^{m} \mathcal{D}_{m} \phi \bar{\eta}^{(1)}+2 \mu^{2} \eta^{(2)} \\
\delta_{\eta} \phi= & \sqrt{2} \chi \eta^{(1)}
\end{aligned}
$$

where one has the relation $m=\kappa \mu^{2}$.

\section{B. Derivation of Supersymmetry Transformations}

In this Appendix we derive the global supersymmetry transformation rules for the massive spin-3/2 and spin-1 multiplets.

\section{B.1 The Massive Spin-3/2 Multiplet}

For the spin-3/2 multiplet we start with the following ansatz for the multiplet's supersymmetry transformation rules, which consists of the most general form permitted by Lorentz invariance and dimensional analysis, provided that any $1 / m$ terms are pure gauge, so that they do not introduce $1 / m$ terms into $\delta \mathcal{L}_{\text {kin }}$. We also require the ansatz to be $U(1)$ invariant where $\mathcal{A}_{m} \rightarrow e^{i \alpha} \mathcal{A}_{m}$ and $\gamma_{L} \varepsilon \rightarrow e^{i \alpha} \gamma_{L} \varepsilon$. This leads to the following form:

$$
\begin{aligned}
\delta \mathcal{A}_{m}= & c_{1}\left(\bar{\eta}_{m} \gamma_{L} \varepsilon\right)+c_{2}\left(\bar{\zeta} \gamma_{m} \gamma_{L} \varepsilon\right)+\frac{c_{3}}{m} \partial_{m}\left(\bar{\zeta} \gamma_{L} \varepsilon\right) \\
\gamma_{L} \delta \zeta= & c_{4} \mathcal{A}_{m n}^{*} \gamma^{m n} \gamma_{L} \varepsilon+c_{5} m \mathcal{A}_{m} \gamma^{m} \gamma_{R} \varepsilon \\
\gamma_{L} \delta \eta_{m}= & \frac{1}{m} \partial_{m}\left(c_{6} \mathcal{A}_{a b}^{*} \gamma^{a b} \gamma_{L} \varepsilon+m c_{7} \mathcal{A}_{a} \gamma^{a} \gamma_{R} \varepsilon\right)+c_{8} \mathcal{A}_{m n} \gamma^{n} \gamma_{R} \varepsilon \\
& +c_{9} \epsilon_{m n a b} \mathcal{A}^{a b} \gamma^{n} \gamma_{R} \varepsilon+c_{10} m \mathcal{A}^{n *} \gamma_{m n} \gamma_{L} \varepsilon+c_{11} m \mathcal{A}_{m}^{*} \gamma_{L} \varepsilon
\end{aligned}
$$


Demanding $\delta \mathcal{L}=0$, order by order in $m$ gives the conditions:

$$
c_{2}=2 c_{4}, \quad c_{3}=-2 c_{4}, \quad c_{5}=-2 c_{4}^{*}
$$

coming from those terms in $\delta \mathcal{L}$ which involve $\zeta$, and the rest give:

$$
c_{6}=\frac{c_{1}}{6}, \quad c_{7}=\frac{2 c_{1}^{*}}{3}, \quad c_{8}=-\frac{2 c_{1}^{*}}{3}, \quad c_{9}=\frac{i c_{1}^{*}}{6}, \quad c_{10}=-\frac{c_{1}}{3}, \quad c_{11}=\frac{2 c_{1}}{3} .
$$

The constants $c_{1}$ and $c_{4}$ are not determined. They could be fixed by closing the algebra and asking the translation generated to be normalized in the standard way. Instead we choose them by comparing with ref. [14], with the idea of making contact with a conventional form for the transformation laws for a later choice of variables. This leads to the choices (keeping in mind that ref. [14 does not use canonically normalized vector fields): $c_{1}=\sqrt{2}$ and $c_{4}=1 / \sqrt{6}$, giving the result quoted in the main text:

$$
\begin{aligned}
\delta \mathcal{A}_{m}= & \sqrt{2}\left(\bar{\eta}_{m} \gamma_{L} \varepsilon\right)+\frac{2}{\sqrt{6}}\left(\bar{\zeta} \gamma_{m} \gamma_{L} \varepsilon\right)-\frac{2}{\sqrt{6} m} \partial_{m}\left(\bar{\zeta} \gamma_{L} \varepsilon\right) \\
\gamma_{L} \delta \zeta= & \frac{1}{\sqrt{6}} \mathcal{A}_{m n}^{*} \gamma^{m n} \gamma_{L} \varepsilon-\frac{2 m}{\sqrt{6}} \mathcal{A}_{m} \gamma^{m} \gamma_{R} \varepsilon \\
\gamma_{L} \delta \eta_{m}= & \frac{1}{m} \partial_{m}\left(\frac{\sqrt{2}}{6} \mathcal{A}_{a b}^{*} \gamma^{a b} \gamma_{L} \varepsilon+\frac{2 \sqrt{2} m}{3} \mathcal{A}_{a} \gamma^{a} \gamma_{R} \varepsilon\right)-\frac{2 \sqrt{2}}{3} \mathcal{A}_{m n} \gamma^{n} \gamma_{R} \varepsilon \\
& +\frac{i \sqrt{2}}{6} \epsilon_{m n a b} \mathcal{A}^{a b} \gamma^{n} \gamma_{R} \varepsilon-\frac{\sqrt{2} m}{3} \mathcal{A}^{n *} \gamma_{m n} \gamma_{L} \varepsilon+\frac{2 \sqrt{2} m}{3} \mathcal{A}_{m}^{*} \gamma_{L} \varepsilon
\end{aligned}
$$

\section{B.2 The Massive Spin-1 Multiplet}

As for the spin-3/2 multiplet described above, the global supersymmetry transformations for the spin-1 multiplet may be obtained by writing down the most general ansatz which is consistent with Lorentz invariance, dimensional analysis and a $U(1)$ symmetry. In this case the $U(1)$ charge is carried by the two fermions, so we suppose the combination $\beta+i \alpha$ rotates by $e^{i \omega}$ (which is the way we assume $\gamma_{R} \varepsilon$ also rotates), so $\beta-i \alpha$ and $\gamma_{L} \varepsilon$ rotate oppositely. The most general transformation becomes:

$$
\begin{aligned}
\delta \varphi & =f_{1} \bar{\varepsilon} \gamma_{L}(\beta+i \alpha)+c . c . \\
\gamma_{L} \delta \beta & =\left(\gamma^{m} \gamma_{R} \varepsilon\right)\left[f_{2} \partial_{m} \varphi+f_{3} v A_{m}\right]+f_{4} v \varphi\left(\gamma_{L} \varepsilon\right)+f_{5} A_{m n}\left(\gamma^{m n} \gamma_{L} \varepsilon\right) \\
\gamma_{L} \delta \alpha & =-i\left(\gamma^{m} \gamma_{R} \varepsilon\right)\left[f_{2} \partial_{m} \varphi+f_{3} v A_{m}\right] \\
& \quad+i f_{4} v \varphi\left(\gamma_{L} \varepsilon\right)+i f_{5} A_{m n}\left(\gamma^{m n} \gamma_{L} \varepsilon\right) \\
\delta A_{m} & =\partial_{m}\left[\frac{f_{6}}{v} \bar{\varepsilon} \gamma_{L}(\beta+i \alpha)\right]+f_{7} \bar{\varepsilon} \gamma_{m} \gamma_{L}(\beta-i \alpha)+c . c .
\end{aligned}
$$

Invariance of the action (up to total derivatives) then implies the relations

$$
f_{1}=f_{2}^{*}=-f_{4}, \quad f_{3}=f_{6}^{*}=f_{7}, \quad f_{7}=-2 f_{5}^{*}
$$


so we can conventionally choose $f_{1}$ and $f_{3}$ to arrange that $\varphi$ has the same transformation rules as does the imaginary part of the scalar in a chiral multiplet. The required choice is $f_{1}=f_{2}=-f_{4}=1 / \sqrt{2}$ and $f_{3}=2 f_{5}=-f_{6}=f_{7}=i / \sqrt{2}$, giving:

$$
\begin{aligned}
& \delta \varphi=\frac{1}{\sqrt{2}} \bar{\varepsilon} \gamma_{L}(\beta+i \alpha)+c . c . \\
& \gamma_{L} \delta \beta= \frac{1}{\sqrt{2}}\left(\gamma^{m} \gamma_{R} \varepsilon\right)\left[\partial_{m} \varphi+i v A_{m}\right]-\frac{v}{\sqrt{2}} \varphi\left(\gamma_{L} \varepsilon\right)+\frac{i}{2 \sqrt{2}} A_{m n}\left(\gamma^{m n} \gamma_{L} \varepsilon\right) \\
& \gamma_{L} \delta \alpha=-\frac{i}{\sqrt{2}}\left(\gamma^{m} \gamma_{R} \varepsilon\right)\left[\partial_{m} \varphi+i v A_{m}\right] i v \\
& \quad-\frac{i v}{\sqrt{2}} \varphi\left(\gamma_{L} \varepsilon\right)-\frac{1}{2 \sqrt{2}} A_{m n}\left(\gamma^{m n} \gamma_{L} \varepsilon\right) \\
& \delta A_{m}=-\partial_{m}\left[\frac{i}{v \sqrt{2}} \bar{\varepsilon} \gamma_{L}(\beta+i \alpha)\right]+\frac{i}{\sqrt{2}} \bar{\varepsilon} \gamma_{m} \gamma_{L}(\beta-i \alpha)+c . c .
\end{aligned}
$$

This is the form quoted in the main text.

\section{References}

[1] J. Polchinski, Phys. Rev. Lett. 75 (1995) 4724.

[2] J. Hughes, J. Liu and J. Polchinski, Phys. Lett. B180 (1986) 370; J. Hughes and J. Polchinski, Nucl. Phys. B278 (1986) 147.

[3] E. Witten, Nucl. Phys. B188 (1981) 513; S. Cecotti, L. Girardello and M. Porrati, Phys. Lett. B145 (1984) 61.

[4] K. Benakli, Phys. Rev. D60, 104002 (1999) hep-ph/9809582; C. P. Burgess, L. E. Ibáñez and F. Quevedo, Phys. Lett. B447, 257 (1999) hep-ph/9810535.

[5] G. Aldazabal, L. E. Ibáñez and F. Quevedo, JHEP 0001 (2000) 031 hep-th/9909172; JHEP 0002 (2000) 015 hep-ph/0001083; G. Aldazabal, L. E. Ibáñez, F. Quevedo and A. M. Uranga, JHEP 0008 (2000) 002 hep-th/0005067; R. Blumenhagen, L. Görlich, B. Körs and D. Lüst, JHEP 0010 (2000) 006 hep-th/0007024; G. Aldazabal, S. Franco, L. E. Ibáñez, R. Rabadán and A. M. Uranga, JHEP 0102 (2001) 047 hep-ph/0011132; J. Math. Phys. 42 (2001) 3103 hep-th/0011073.

[6] C. P. Burgess, R. C. Myers and F. Quevedo, Phys. Lett. B495 (2000) 384, hep-th/9911164.

[7] L. Randall and R. Sundrum, Nucl. Phys. B557 (1999) 79, hep-th/9810155.

[8] G. Giudice, M. A. Luty, H. Murayama and R. Rattazzi, JHEP 9812 (1998) 027 hep-ph/9810442]; J. Bagger, T. Moroi and E. Poppitz, JHEP 0004 (2000) 009 hep-th/9911029; M. K. Gaillard and B. Nelson, Nucl. Phys. B588 (2000) 197 hep-th/0004170. 
[9] A. Anisimov, M. Dine, M. Graesser and S. Thomas, hep-th/0201256.

[10] E. Dudas and J. Mourad,Phys. Lett. B514 (2001) 173 hep-th/0012071 J. Bagger, F. Feruglio and F. Zwirner, JHEP 0202 (2002) 010 hep-th/0108010; I. Antoniadis, K. Benakli and A. Laugier, hep-th/0111209]; K. A. Meissner, H. P. Nilles and M. Olechowski, hep-th/0205166]; M. Klein, hep-th/0205300.

[11] D. Marti and A. Pomarol, Phys. Rev. D64 (2001) 105025 hep-th/0106256; D. E. Kaplan and N. Weiner, hep-ph/0108001.

[12] For early discussions of supersymmetry breaking due to branes see E. Kiritsis and C. Kounnas, Nucl. Phys. B503 (1997) 117 hep-th/9703059; T. Banks and M. Dine, Nucl. Phys. B479 (1996) 173 hep-th/9605136]; E. Dudas and C. Grojean, Nucl. Phys. B507 (1997) 553 [hep-th/9704177]; I. Antoniadis and M. Quirós, Phys. Lett. B416 (1998) 327 hep-th/9707208; Z. Lalak and S. Thomas, Nucl. Phys. B515 (1998) 55 hep-th/9707223; E. Dudas, Phys. Lett. B416 (1998) 309 hep-th/9709043; H. P. Nilles, M. Olechowski and M. Yamaguchi, Phys. Lett. B415 (1997) 24 hep-th/9707143; T. Li, J. Lopez and D. Nanopoulos, Mod. Phys. Lett. A12 (1997) 2647 hep-ph/9702237; K. Choi, Phys. Rev. D56 (1997) 6588 hep-th/9706171]; K. Choi, H. B. Kim and C. Muñoz, Phys. Rev. D57 (1998) 7521 [hep-th/9711158]; A. Lukas, B. Ovrut and D. Waldram, Phys. Rev. D57 (1998) 7529 hep-th/9711197]; Nucl. Phys. B532 (1998) 43 hep-th/9710208; E. Mirabelli and M. Peskin, Phys. Rev. D58 (1998) 065002 hep-th/97065002.

[13] Nonlinear realizations associated with partial supersymmetry breaking are discussed by: S. Samuel and J. Wess, Nucl. Phys. B221 (1983) 153; J. Bagger and J. Wess, Phys. Lett. B138 (1984) 105; J. Bagger and A. Galperin, Phys. Lett. B336 (1994) 25 hep-th/9406217; Phys. Rev. D55 (1997) 1091 hep-th/9608177; I. Antoniadis, H. Partouche and T. R. Taylor, Phys. Lett. B372 (1996) 83 hep-th/9512006;

S. Ferrara, L. Girardello and M. Poratti, Phys. Lett. B376 (1996) 275 hep-th/9512180; J. Bagger, Nucl. Phys. Proc. Suppl. 52A (1997) 362 hep-th/9610022; J. Louis, hep-th/0203138.

[14] R. Altendorfer and J. Bagger, in Particles, strings and cosmology, Boston (1998) 742 hep-th/9809171]; Phys. Lett. B460 (1999) 127 hep-th/9904213.

[15] S. Ferrara and P. van Nieuwenhuizen, Phys. Lett. B127 (1983) 70.

[16] C. P. Burgess and D. London, Phys. Rev. Lett. 69 (1992) 3428; Phys. Rev. D48 (1993) 4337 hep-ph/9203216.

[17] J. M. Cornwall, D. N. Levin and G. Tiktopoulos, Phys. Rev. D 10, 1145 (1974) [Erratum-ibid. D 11, 972 (1975)]; M. S. Chanowitz, M. Golden and H. Georgi, Phys. Rev. D 36, 1490 (1987); M. S. Chanowitz, Ann. Rev. Nucl. Part. Sci. 38, 323 (1988).

[18] We follow the conventions of S. Weinberg, The Quantum Theory of Fields, Vol III, Cambridge University Press (2000). 
[19] P. Van Nieuwenhuizen, Phys. Rept. 68 (1981) 189.

[20] J. P. Derendinger, S. Ferrara, A. Masiero and A. Van Proeyen, Phys. Lett. B140 (1984) 307.

[21] D. Cremades, L. E. Ibáñez and F. Marchesano, JHEP 0207 (2002) 009 hep-th/0201205.

[22] P. West, Introduction to Supersymmetry and Supergravity, World Scientific (1986).

[23] C. W. Misner, K. S. Thorne and J. A. Wheeler, Gravitation, W. H. Freeman, San Francisco (1970).

[24] J. Wess, J. Bagger, "Supersymmetry and Supergravity", 2nd ed., Princeton University Press (1992). 